\title{
Closed-Loop Brain Model of Neocortical Information-Based Exchange
}

\author{
James Kozloski * \\ IBM Research Division, Computational Biology Center, IBM T.J. Watson Research Center, Yorktown Heights, NY, USA
}

Here we describe an "information-based exchange" model of brain function that ascribes to neocortex, basal ganglia, and thalamus distinct network functions. The model allows us to analyze whole brain system set point measures, such as the rate and heterogeneity of transitions in striatum and neocortex, in the context of neuromodulation and other perturbations. Our closed-loop model is grounded in neuroanatomical observations, proposing a novel "Grand Loop" through neocortex, and invokes different forms of plasticity at specific tissue interfaces and their principle cell synapses to achieve these transitions. By implementing a system for maximum information-based exchange of action potentials between modeled neocortical areas, we observe changes to these measures in simulation. We hypothesize that similar dynamic set points and modulations exist in the brain's resting state activity, and that different modifications to informationbased exchange may shift the risk profile of different component tissues, resulting in different neurodegenerative diseases. This model is targeted for further development

OPEN ACCESS

Edited by:

Wolfram Schenck, University of Applied Sciences Bielefeld, Germany

Reviewed by: Alino Martinez-Marcos, Universidad de Castilla, Spain Alexander Peyser, Forschungszentrum Jülich, Germany

*Correspondence:

James Kozloski kozloski@us.ibm.com

Received: 29 August 2015 Accepted: 02 January 2016 Published: 18 January 2016

Citation:

Kozloski J (2016) Closed-Loop Brain Model of Neocortical Information-Based Exchange.

Front. Neuroanat. 10:3. doi: 10.3389/fnana.2016.00003 using IBM's Neural Tissue Simulator, which allows scalable elaboration of networks, tissues, and their neural and synaptic components toward ever greater complexity and biological realism.

\section{Keywords: neocortex, thalamus, basal ganglia, information-based exchange, brain model}

\section{INTRODUCTION}

Synaptic plasticity regulates neuronal responses to patterns of inputs impinging on dendritic arbors from multiple presynaptic sources. Resulting input selectivity at the single neuron level is often associated with learning and memory in models of cognition. At the circuit level, synaptic plasticity can serve more complex functions over arbitrary inputs, from selecting fixed points in recurrent networks (Hopfield, 1982), to implementing optimizations such as information maximization in artificial neural networks (Linsker, 1997), to dynamically encoding inputs in winnerless networks (Rabinovich et al., 2001). A challenge to analyzing the role of any neuron or circuit that implements these functions for cognition is that of modeling appropriate, naturalistic neuronal and circuit inputs, which in real brains derive from tens of thousands to millions of other neurons.

Here we present a closed-loop brain model, including component models of several neural tissues that we hypothesize implement some of these functions. Synapses and plasticity connecting components at principle cell interfaces together create a set of closed neuroanatomical loops. Without extrinsic inputs or stochastic intrinsic drivers, our model avoids the challenges and 
assumptions of modeling naturalistic inputs separately, and instead derives them exclusively from the dynamics of upstream neurons and tissues. The challenge then is model validation, which we won't address in this report. Instead the aim here is to delineate hypotheses and a theory of brain resting state function using the model and its simulations. We propose that models implemented similarly constitute a class of "brain models", and are distinct from component "neural tissue models," which instead assume an arbitrary set of inputs or stochastic processes to drive intrinsic tissue dynamics. By avoiding these assumptions, a coarse but consistent model of global brain function may be useful for better constraining the most detailed neural tissue simulations.

We introduce the term "traversal" to refer to a "synfire chain" as defined by Abeles (1991), but with additional neuroanatomical constraints defining a minimum set of neocortical regions traversed by the event. The cortico-cortical feedback loop in our model acts as a substrate for combined traversals of sensory, limbic, and motor areas, which we propose together drive behavior in the organism. The cortico-thalamocortical feed forward loop acts to maximize the entropy of these global traversals and to maximize information about the environment relayed as inputs to the loop. Lastly, the striato-nigro-striatal loop provides a means to select subsequent configurations by monitoring changes in ongoing traversals and signaling them with dopamine to alter routing within the feed forward entropy maximizing network. We propose this function as the substrate for reward learning in the organism. Each of these loops therefore has both a closed-loop function (global traversal, traversal entropy maximization, and traversal change monitoring and rerouting) and an organismal input-output function (behavior generation, sensory processing, and behavior selection based on reward learning).

The objective of this report is to describe the closedloop model and simulations of it. Perturbations to the closed loop that alter dynamic set points will also be described. We hypothesize about dynamic disease mechanisms and progression based on the model, and describe methods that use neural tissue simulation (Kozloski and Wagner, 2011) for modeling treatments that alter brain disease risks. To summarize our overall approach and long-term research goals, the driving hypotheses relating our closed-loop model to brain disorder and disease states are: (1) The primary disease and disorder risk is a disturbance in plasticity that critically maintains brain system dynamic set points; (2) Compensatory circuit dynamics achieves near-normal set points despite genetic or environmental perturbations, but with increased secondary risk of neuronal dysfunction, damage, or loss; (3) Secondary risk correlates with feed forward destruction or dysfunction of neural tissues because with each neuron function lost, maintaining system set points requires even greater secondary risks; and (4) Slowing progression may therefore lie in mitigating the primary risk's effect on system set points or in limiting secondary risks incurred by inherent compensatory dynamics.

\section{INFORMATION-BASED EXCHANGE BRAIN MODEL}

\subsection{Cytoarchitectonics of Bidirectional Neocortical Projections: The "Grand Loop"}

We propose a model that emphasizes a specific cortico-cortical connectivity across the major sensory, limbic, and motor categories of Brodmann areas. This emphasis derives from several observations. First, we note the importance of signals traversing all three categories of cortical representations in order to produce a stable basis for perception and behavior by integrating information about the environment, internal needs, and behavioral opportunities of the organism. While many loops have been discovered in studies of the neocortical connectome, none provide the directed graph (feed forward vs. feedback) needed to identify a system to support such traversals. Instead, we note that the cytoarchitectonic granularity of neocortical areas provides one means to interpret feed forward (i.e., more granular to less granular) and feedback (i.e., less granular to more granular) connections between cortical areas (Rempel-Clower and Barbas, 2000) and therefore a means to identify a backbone for global brain traversals (Figure 1A).

Granularity refers to the density of punctate Nissl bodies in stained layer 4 of neocortex. The granularity across all of neocortex was studied and mapped extensively by von Economo (1929), and we reproduce his illustrations and some key findings in Figures 1A,B. Note that granular cortices typically have smaller diffuse Nissl bodies in layer 5, and agranular cortices have very large diffuse layer 5 Nissl bodies. Tiling in von Economo's map shows that regions of cortex with similar granularity are adjacent, with key exceptions at the boundaries between primary motor (M1) and primary somatosensory (S1), hippocampus (HC) and retrosplenial granular areas (RGA), and subgenual anterior cingulate (ACC) and prefrontal (PFC) cortices. Each of these three pairs of Brodmann areas are interconnected, and in our model represent key boundaries in the backbone for traversing the sensory-limbic (HC-RGA), limbic-motor (ACC$\mathrm{PFC}$ ), and motor-sensory (M1-S1) cortices (Figure 1B, black arrows). To complete a "Grand Loop" backbone, we join each pair of areas by an area in their adjacent dysgranular neocortical regions: the secondary somatosensory (S2), posterior cingulate (PCC), and supplemental motor (SMA) areas (Figure 1C). While others have noted that organizing principles for intrinsic microcircuits may be derived from combining von Economo's observations with those regarding granularity and the direction of cortico-cortical projections (Beul and Hilgetag, 2015), none to our knowledge have proposed a Grand Loop that traverses all of neocortex according to these principles.

\subsection{Cortico-Cortical and Cortico-Thalamo-Cortical Functional Pathways}

Having defined the feed forward neocortical Grand Loop, we'll now embellish this structural model with additional components based on observations regarding feed forward projections and 

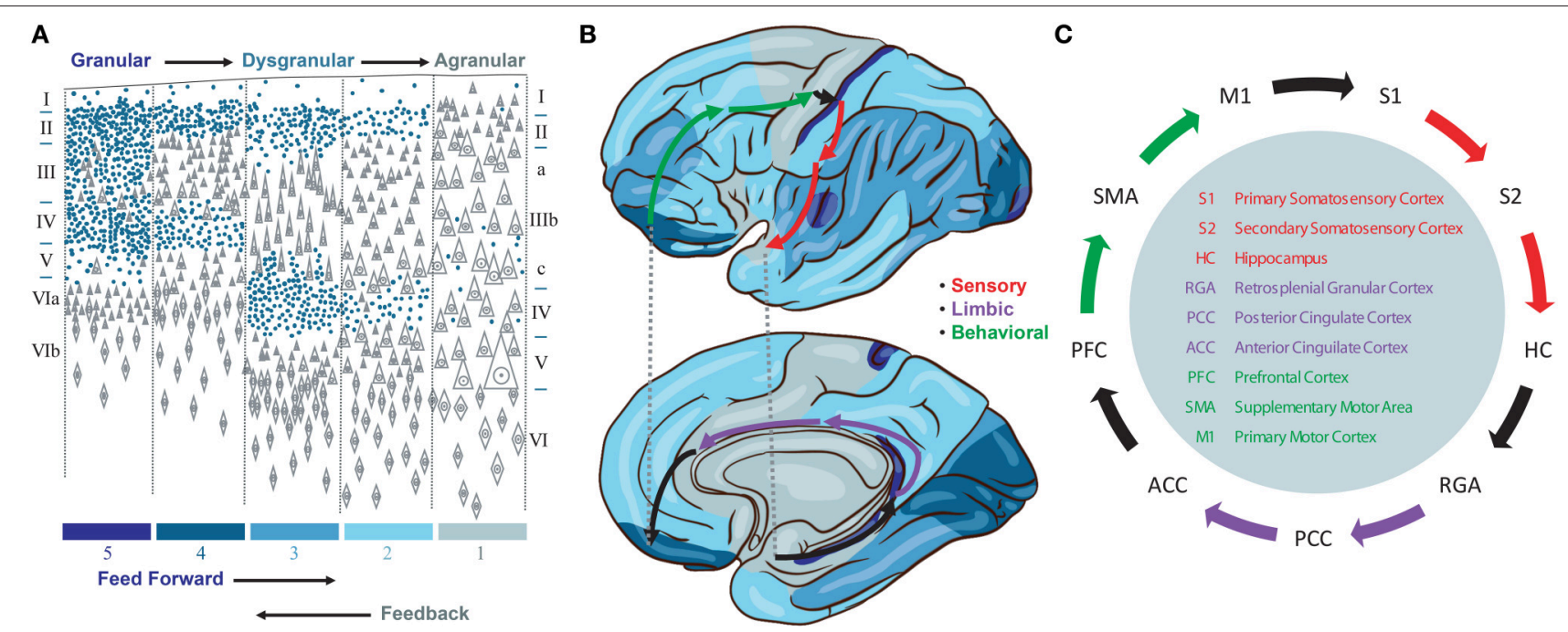

FIGURE 1 | (A) Granularity of different neorcortical areas, adapted from von Economo (1929). Colors at bottom correspond to the map in (B). (B) von Economo's neocortical tiling based on the granularity of large regions of neocortex spanning multiple Brodmann areas. The location of three Brodmann areas per stage are waypoints along a feed forward Grand Loop (arrows). (C) These Brodmann areas are connected based on projection data. Evidence that feed forward connections progress from granular to agranular areas provides directionality. The reciprocal feedback loop is not shown.

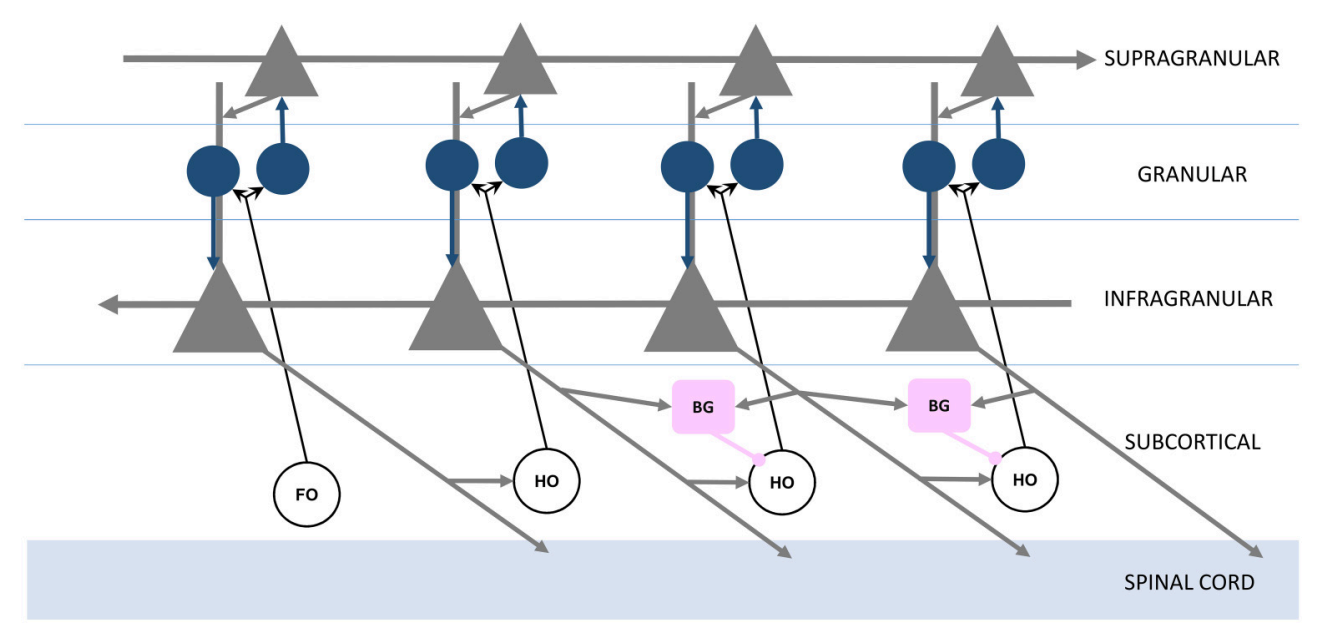

FIGURE 2 | Organization of feed forward and feedback functional connections, adapted from Guillery and Sherman (2011). Infragranular and supragranular layer pyramidal neurons (gray triangles) form direct feedback and feed forward connections, with the local circuitry receiving first order (FO) and higher order (HO) thalamic nuclei inputs through granular layer spiny stellate neurons (blue circles). The basal ganglia (BG, pink boxes) receive infragranular inputs, and provide inhibitory gating to higher order nuclei in the brain's frontal lobe (right).

signaling between neocortical areas. Sherman and Guillery emphasized different roles for direct cortico-cortical feed forward projections, which join one cortical area to another through their supragranular layers, and indirect cortico-thalamo-cortical projections, which join infragranular layers of the same original area to the granular layer of the same target area (Figure 2; Guillery and Sherman, 2011). In Sherman and Guillery's model, direct cortico-cortical projections are "modulatory," providing restricted activation to the target area, and indirect corticothalamo-cortical projections are "driving," providing activation across all layers of the target area. Figure 2 represents Sherman and Guillery's model (based on a simplification of their schematic). We will now describe how this model may be integrated into the Grand Loop.

Recall that each station of the loop in Figure $\mathbf{1}$ is coupled in the feed forward direction. These connections, largely through the supragranular layers, are mirrored in the feedback direction by connections through infragranular layers (Figure 2; RempelClower and Barbas, 2000). Thus, the Grand Loop represents two reciprocal loops, one in the feed forward direction and one in 
the feedback direction. Furthermore, according to Sherman and Guillery, higher order thalamic nuclei provide at every stage a redundant relay for driving inputs over each feed forward connection. The local cortical circuit then receives signals from these thalamic nuclei and mixes the otherwise independent direct feed forward modulation loop and feedback traversal loop, primarily at layer 4's synaptic connections onto supragranular layers, and at supranular layers' onto the infragranular layers' apical dendrites.

We proposed previously that layers $2 / 3$ of neocortex implement a network for maximizing mutual information between thalamic inputs and cortical responses (Kozloski et al., 2007). Entropy maximization in these layers (equivalent to information maximization when noise in the inputs is assumed to be negligible) would require a dense lateral network (Linsker, 1997), which fits well with the high proportion $(\sim 22 \%)$ of total cortical synapses dedicated to intralaminar $2 / 3$ connections (Binzegger et al., 2004). Given this role for the supragranular layers, we now propose that the role of corticothalamo-cortical driving inputs in Sherman and Guillery's model is to provide inputs both from first order thalamic nuclei about the environment and from feedback traversals through higher order thalamic nuclei about the behavioral state of the organism. A global supragranular network then extracts maximally informative features from combinations of these inputs. In addition, we propose that these features become conditional modulators on feedback traversals by boosting or reducing the gain on proximal inputs to layer 5 neurons by means of synaptic inputs onto their apical dendrites from layer $2 / 3$ neurons.

\subsection{Basal Ganglia Gating of Feed Forward Functional Pathways}

In Sherman and Guillery's model, thalamic relay neurons in both first order and second order nuclei are subject to modulation. Modulation may derive from direct cortico-thalamic feedback from layer 6, inhibition from the thalamic reticular nucleus, or from neuromodulatory inputs such as norepinepherine from the locus coeruleus. Sherman and Guillery's model derives largely from their studies of sensory cortices and feed forward pathways through them, projecting from more granular to less granular regions. Here we extend the discussion of thalamic relay neuron modulation to include a role for inhibitory inputs from the basal ganglia to thalamic nuclei that act as relays in the frontal lobe between more granular limbic and motor areas to less granular areas in these regions.

The basal ganglia (including ventral limbic and dorsal motor) are in a privileged position to influence traversals by means of their inhibitory inputs onto thalamic relay neurons within the Grand Loop. These inputs derive from nucleus inominata in the ventral limbic subpallium and from the globus pallidus in the dorsal motor subpallium. Neurons in the ventral pallidus (nucleus inominata) receive inhibition from medium spiny neurons (MSNs) in the nucleus accumbens (ventral striatum) and those in the dorsal globus pallidus from those in the dorsal striatum. These neurons then either directly disinhibit thalamic relay neurons or indirectly inhibit thalamic relay neurons through an additional stage of inhibitory neurons (in globus pallidus, this is organized as direct and indirect projections through the external and internal segments). Spiking models of inhibitory pallidothalamic gating have focused on the bird song system (Goldberg et al., 2012), where gating inputs to thalamic relay neurons serve the role of transitioning syllables of the organism's vocalizations. Here we propose a more generic role for this gating in selecting and deselecting different pathways for internal traversals of the pallium.

Inputs to these direct and indirect pathways through basal ganglia derive from neocortical layer 5 neurons' projections onto MSNs, and their corticostriatal synapses undergo spiketiming dependendent plasticity (STDP) which is modulated differentially by dopamine depending on the selective expression of either D1 dopamine receptors in the direct or D2 dopamine receptors in the indirect pathways (Pawlak and Kerr, 2008; Figure 3). Each layer 5 neuron's collaterals then include a branch descending to the brainstem or spinal cord, a branch descending to thalamus (Guillery and Sherman, 2011), and additionally a branch descending to striatum (Lévesque et al., 1996). A recent review of additional types of layer 5 projection neurons and the role of corticostriatal connectivity in disease provides a thorough examination and schematic of these pathways (Shepherd, 2013), and our model of thalamic gating, for now and for simplicity, includes only the "Pyramidal Tract" layer 5 neurons and their projections to basal ganglia and thalamus for the function of thalamic gating.

In summary, our model extends Sherman and Guillery's model of cortico-thalamo-cortical gating of driving, feed forward inputs to include modulation from striatal and pallidal neurons in both the direct and indirect pathways (Figure 3). MSNs in our model receive convergent layer 5 collaterals from all layer 5 neurons that send convergent collaterals onto a specific thalamic relay neuron. This relay neuron is then gated by the same MSNs, indirectly through globus pallidus (the gate opens for the direct pathway, and closes for the indirect pathway). Such a scheme does not preclude so called "closed loops" that originate and terminate in the same cortical area (Kelly and Strick, 2004), but downplays their significance as only partial regulators of feed forward thalamic gating (Figure 4A).

The basal ganglia in our model is then a "forward driver gate" for all feed forward driving signals relayed through the frontal lobe's cortico-thalamo-cortical functional pathways. Because these pathways relay layer 5 traversals through thalamus to the granular and supragranular layers of cortex, they can indirectly control the routing of feedback signals and the selection of certain traversals over others through the Grand Loop, as we describe in the next section. Additional area to area corticothalamo-cortical pathways not on the main loop backbone (such as the visual system) are then available for additional modulation and traversals of the global layer 5 behavioral network, possibly including loops requiring reafference from the environment.

\subsection{Information Based Gating of Feedback Traversals}

Our model provides two distinct functional signaling pathways through the Grand Loop: feed forward for driving the 


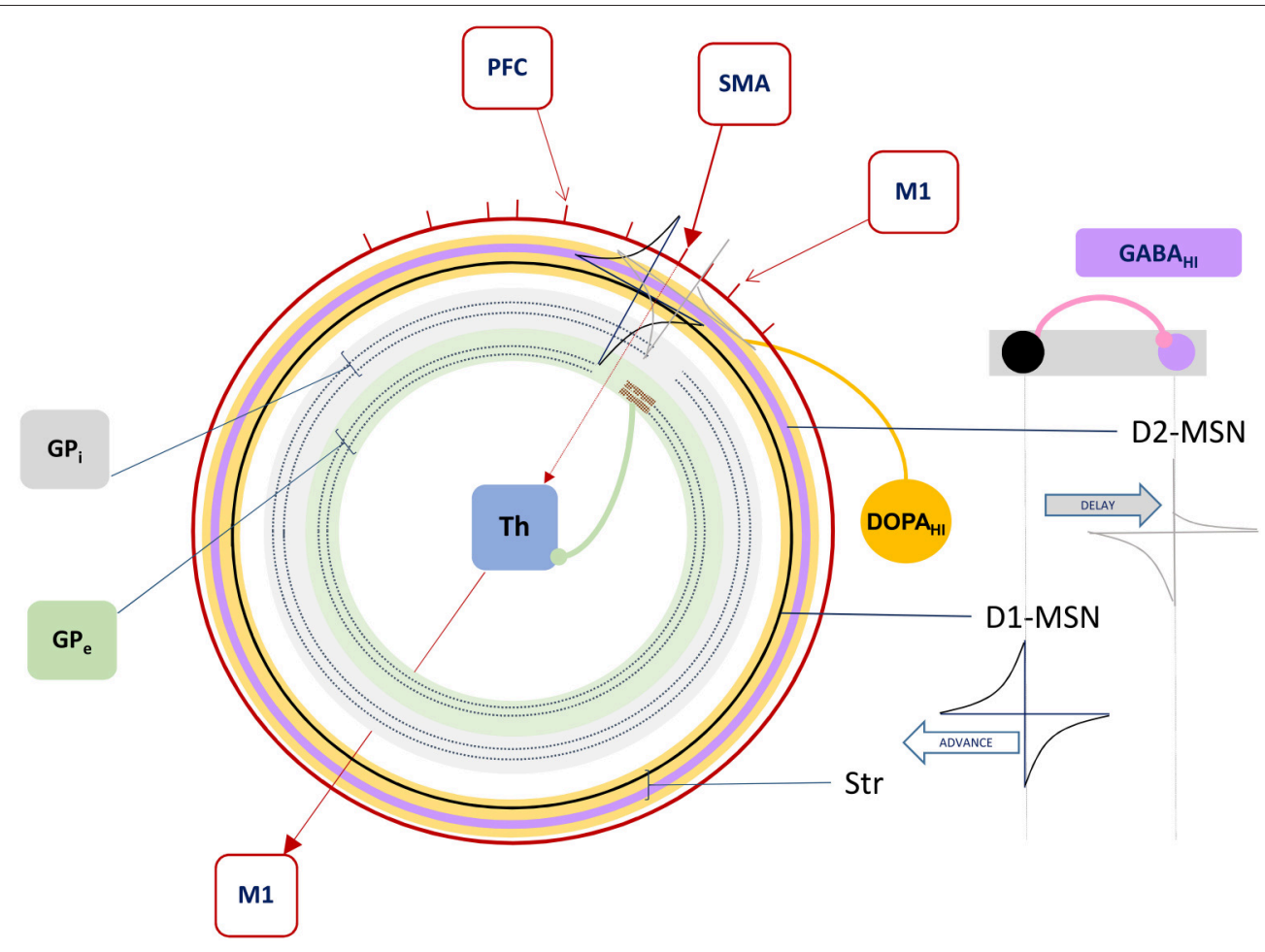

FIGURE 3 | The Forward Driver Gate. Cortical action potentials (red raster marks) traverse the Grand Loop (red circle, representing a periodic time line), when neurons in specific areas (red boxes) spike. These cause spikes in striatum, represented by STDP functions placed on a periodic time line for both the indirect (lavender circle) and direct (black circle) pathway medium spiny neurons (D2-MSN and D1-MSN). The D1-MSN is inhibiting the D2-MSN providing additional GABA-ergic modulation of STDP. Spikes cause direct disinhibition of the external segment of Globus Pallidus (GPe), allowing a cortical spike to be relayed through the thalamic gate (red arrows, SMA to M1), or indirect additional inhibition through the internal segment (GP $)$, blocking spikes.

supragranular entropy maximizing network, and feedback for traversal of the infragranular behavior generation network. The latter, in our implementation of the model, propagates synfire events through a loop, as described by Zheng and Triesch in their model of "synfire ring" formation and propagation (Zheng and Triesch, 2014). Restricting synfire activity to the feedback direction is a key aspect of our model. Unlike other models of feedback, which ascribe to it solely a sensory processing "top down" function, we model the propagation of feedback activity as potentially independent of feed forward activity (for example when a coupling parameter between these two networks is zero). Specifically, the emergence of activations in the supragranular layers are rate coded, while activations in the infragranular layers are spike timing based in order to support synfire events. (We won't speculate here on how these distinct coding schemes are implemented and maintained by the neocortical microcicuit, but it would seem there are ample mechanisms available.)

Conditional coupling between features, extracted by information maximization in the supragranular layers, and spike propagation in the infragranular layers, is then under the control of a parameter that models cholinergic modulation in neocortex. Acetylcholine enhances the influence of sensory inputs on pyramidal cell firing relative to their processing of intrinsic signals within neocortical circuits (Hasselmo and
Giocomo, 2006). We model this modulatory parameter as changing the slope and dynamic range of a gain function. The function sets the gain on feedback integration within the synfire ring based on the level of activity in the corresponding functional units (e.g., orientation columns) in layer $2 / 3$. Thus, feature encoding acts as a gate for synfire propagation, and we hypothesize this gain function may be implemented by layer $2 / 3$ inputs to layer 5 neurons' apical dendrites. Varying cholinergic modulation of these inputs in cortex then controls the slope and dynamic range of the mapping from layer $2 / 3$ activity to layer 5 feedback integration gain. The result is that propagation of synfire activity through a column of cortex is informed by the categorization of thalamic inputs to that same area. Information maximization among responses in the supragranular areas over environmental inputs becomes entropy maximization of synfire propagation pathways through the infragranular layers, provided that coupling between these is strong (i.e., cholinergic modulation is high). It is because of this coupling that we have named our model an information-based exchange network.

\subsection{The Forward Driver Gate: Bursting, Modulation, and Plasticity}

Having proposed a central cortico-thalamo-cortical routing function for striatal MSNs by means of their directly disinhibiting 
A

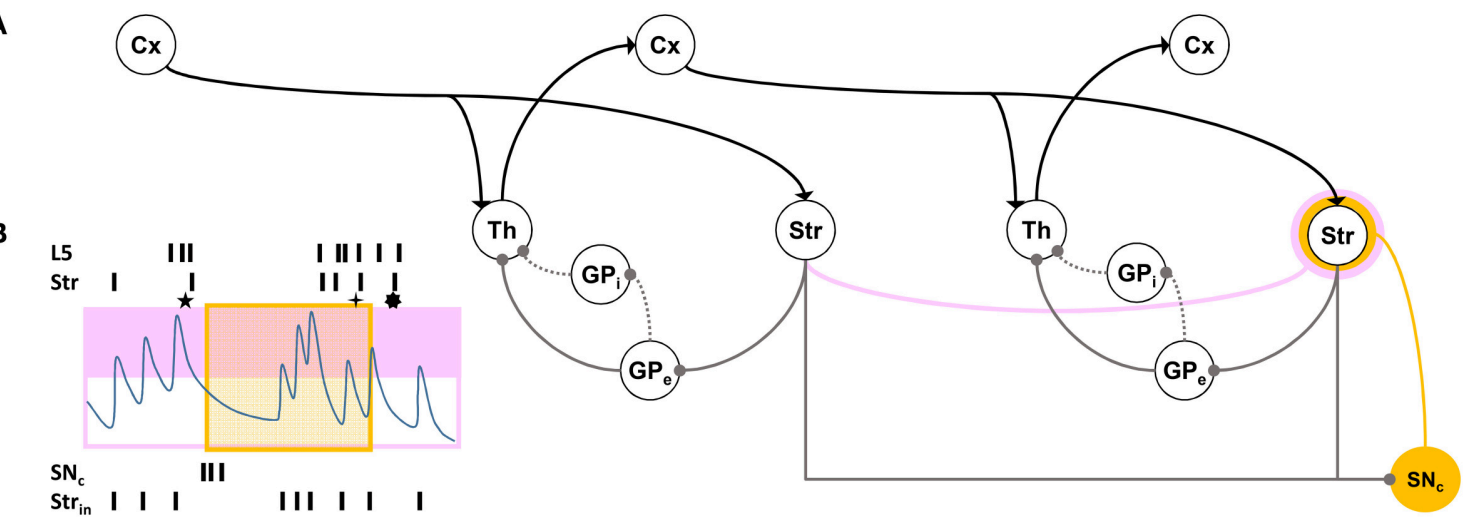

c

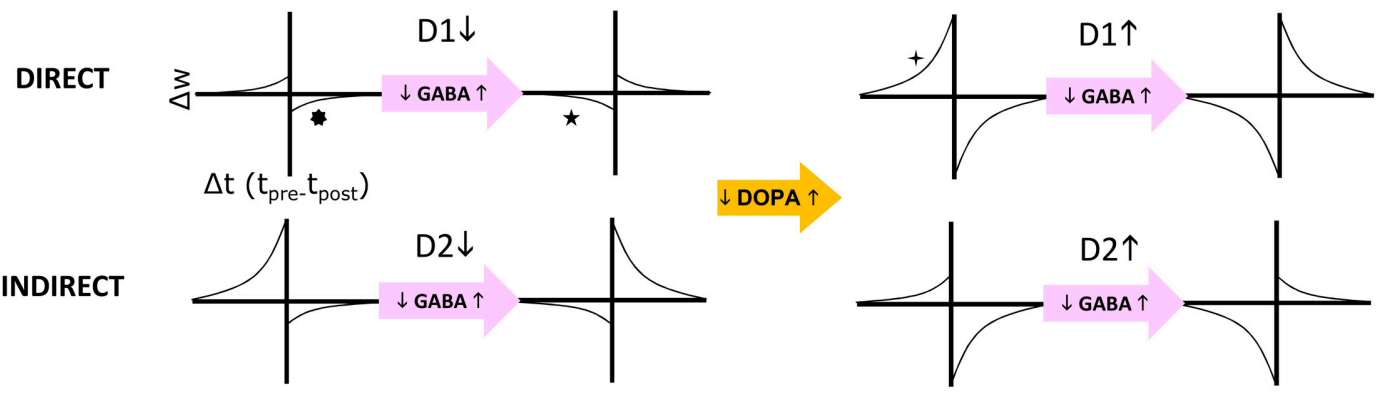

FIGURE 4 | (A) Schematic of cortico-thalamo-cortical routing. Direct (solid line) and indirect (dotted line) pathways through GP disinhibit or inhibit thalamic relay neurons. The striatum is a source of self inhibition (pink line) creating GABA-ergic modulation of corticostriatal synapses (pink annulus). Dopamine neurons in SNc receive extensive inhibitory striatal inputs, and similarly modulate these synapses (yellow annulus). (B) GABA levels change with intrinsic striatal firing Str in and cross a threshold (pink box). GABA modulation results in changes to STDP due to correlated layer 5 (L5) and striatal (Str) firing patterns. Superimposed dopamine modulation (yellow box), results in distinctly different STDP functions at a corticostriatal synapse (stars, corresponding to those in C). (C) For each combination of a direct pathway D1 or indirect pathway D2 (rows) striatal neuron's modulatory inputs, model STDP functions are represented. Transitions from low dopamine to high dopamine occurs from left half to right half of this matrix of functions. Transitions from low GABA to high GABA occurs between odd and even columns in the matrix.

or indirectly inhibiting thalamic relay neurons, we will now propose on what basis a striatal MSN adapts to perform this function in the context of system set points. We call this the forward driver gate's "routing function." Our model of MSN firing includes constraints from a weak, assymetric lateral inhibitory network giving rise to "winnerless competition" (Rabinovich et al., 2001), and closely matching the periods of striatal bursting lasting hundreds of milliseconds observed in vivo (Miller et al., 2008). Ponzi and Wickens have similarly used this network to model spiking properties of striatum (Ponzi and Wickens, 2010), and have shown that at transition points in the lateral network configuration (from low, $\sim 10 \%$, to high, $\sim 20 \%$, rates of connectivity), an optimal balance is achieved that facilitates winnerless encoding of variations in driving inputs from neocortex (Ponzi and Wickens, 2013). To achieve this balance, our model instead varies the strength of cortical inputs dynamically by a dual source of modulation of STDP at the corticostriatal synapse.

The first dynamic modulator of STDP at the corticostriatal synapse in our model is GABA inhibition from the lateral network, itself responsible for "turn-taking" among MSNs and their bursts, characteristic of the winnerless network. We assume that both direct and indirect pathways show STPD reversal under
GABA inhibition (Fino et al., 2010; Paille et al., 2013), and we model winnerless competition between striatal neurons as the source for this inhibition (Figure 4B).

The second dynamic modulator of STDP at the corticostriatal synapse is dopamine. Given the routing function's potential as a critical determiner of the emergence of behavior, affect, and cognition in the organism via its direct control over traversals of the layer 5 network, reward-based learning of this function is ultimately required. For now, we simulate our brain model of information-based exchange with dopaminebased learning serving only a closed-loop function, separate from the environment and therefore independent of reward encoding. This closed-loop function is sensitive to system set points and monitors traversals. It is equivalent to so-called "tonic firing" in dopamine neurons, which can also include bursts. We propose that the intrinsic dynamics of dopamine neuron membrane currents implements this closed-loop function by measuring time and the abruptness of changes to system states, with bursts generated under specific conditions summarized below. Dopamine provides a potent modulation of STDP at the corticostriatal synapse (Pawlak and Kerr, 2008), and further modulates it differentially at the inputs to D1-MSNs and D2MSNs. In our model this differential modulation, combined 
with GABA modulation, produces the complex routing function summarized in Figure 4C.

Dopamine neurons have been shown to fire bursts of action potentials to signal basal reward inputs to the organism encoded as strong excitatory inputs to medial tegmentum and substantia nigra pars compacta ( $\mathrm{SNc}$ ) neurons. Because of these responses, the dopamine system has been extensively modeled as recapitulating reinforcement learning and operant conditioning in the organism. We propose here for the first time an additional closed-loop role for dopamine neurons in learning routing functions and selecting traversals. Specifically, we propose that dopamine neurons signal changes to traversals, and thereby influence the subsequent emergence of new traversals. The basis for this proposal derives from recent connectomics studies, which demonstrate that $70 \%$ of dopamine neuron inputs are inhibitory, and that most of this inhibition arises from striatum (Watabe-Uchida et al., 2012). Our closed-loop role for dopamine modulation depends on this inhibition, and this proportion and source suggests that closed-loop responses to inhibitory inputs, not open loop responses to basal excitation and reward, may be the predominant operating mode of the dopaminergic system.

Dopamine neurons exhibit heterogeneous combinations of intrinsic $I_{h}$ and $I_{A}$ currents (Amendola et al., 2012), as well as T-type calcium currents, which together generate post inhibitory rebound bursting in slice preparations. These currents' role in vivo has not yet been demonstrated, but our model assumes that the dynamical criteria for dopamine neuron bursting (and subsequent learning of routing at corticostriatal synapses) are implemented at least in part by rebound bursting. Other models have explored rebound bursting in dopamine neurons (Lobb et al., 2011), but not in the context of a closed-loop regulatory function. In our model, if the duration and abruptness of removal of striatal inhibition to dopamine neurons is appropriate, a rebound burst occurs. This aspect of the model indirectly imposes the additional criterion that inputs from layer 5 to striatum that transition the MSN winnerless network should be similarly matched to the duration and abruptness of change required for rebound spiking in dopamine neurons. In this way the striatonigro-striatal loop monitors changes in traversals and alters routing within the feed forward entropy maximizing network by modulating corticostriatal STDP. We therefore propose that MSNs learn this routing based in part on their ability to recognize patterns of spiking in layer 5 that remain stable for a minimum duration of time then fade rapidly, a property expected during traversals of the Grand Loop.

\section{SIMULATION METHODS}

We simulated the model to explore its dynamics, characterize preliminary set points for measurement and analysis, and study traversal behavior and its regulation under different modulatory conditions. The five major components of the model to be simulated included cortical layers $2 / 3,5$, thalamus, striatum, and dopamine neurons. Meeting this challenge at the detailed level of neural tissue simulation is beyond the scope of this report, and without a good understanding of target model set points, likely impossible. We therefore aimed to draw upon four simplified abstractions of the key behaviors we ascribe to principle cells in these structures (Linsker, 1997; Rabinovich et al., 2001; Mihalas and Niebur, 2009; Zheng and Triesch, 2014). With four base component models replicated from other studies, we then coupled them across novel interfaces, realizing the closed, functioning Grand Loop, complete with its subcortical regulators.

\subsection{Component Models}

Four component models from the literature were targeted here to capture the functions of cortical layers $2 / 3$ and 5 , striatum, and dopamine neurons in the brain model. These four met sufficient requirements to implement informationbased exchange (Algorithm 1), with very few changes to published parameters. We list the models below and describe the requirements they satisfy. Parameters defined in the original references for each component model are found in Table 1. Because thalamic relay neurons were implemented as a simple set of sums over inputs, they are described as an interface between component models in the subsequent section.

- Neocortex, layer 2/3: The model applies the "Infomax" algorithm of Bell and Sejnowski (1995) to thalamic relay neuron inputs. A neural network implementation of the same optimization (Linsker, 1997), based entirely on a local learning rule, establishes the biological plausibility of this function for this tissue (Kozloski et al., 2007). The three stage network modifies a weight matrix $C$ that couples thalamic relay neurons to cortical units (e.g., at layer 4 ) based on microcircuit feedback from layer $2 / 3$. Stage one receives the rate vector $\widehat{x}_{I}$ from an ensemble of time averaged thalamic spike trains and computes the zero mean input vector $x_{I}=\widehat{x}_{I}-x_{0}$, where $x_{0} \approx\left\langle\widehat{x}_{I}\right\rangle$ is learned at the learning rate $\beta_{x_{0}}$. Stage two computes the sum of weighted inputs to stage three, $u \equiv C x_{I}$. In addition, each stage two unit computes an element of the output vector $y$, $y_{i}=\sigma\left(u_{i}\right)$, where $\sigma(\cdot)$ denotes a nonlinear squashing function, here the logistic transfer function, $y=1 / 1+e^{-\left(u+w_{0}\right)}$, where $w_{0}$ is an adaptive output bias vector learned by $\Delta w_{0}=\beta_{w_{0}}[1-$ $2 y$ ]. The output vector $y$ maximizes the mutual information over the input ensemble and provides regulatory microcircuit feedback to the model of layer 5 described below.

Stage three then computes an entropy maximizing learning vector, which is fed back to stage two and applied by Hebbian learning to modify $C$ at the learning rate $\beta_{C_{0}}$. Derived by Linsker (1997), this learning vector when applied in this way precisely yields the Infomax anti-redundancy term of Bell and Sejnowski (1995), $\left(C^{\prime}\right)^{-1}$ (ie., the inverse of the transpose of the input weight matrix) which for simplicity may also be computed directly. In this model of layer $2 / 3$, the entropy maximizing learning vector emerges from a fully connected lateral network, whose weight matrix $\widehat{Q}$ undergoes Hebbian learning according to $\Delta \widehat{Q}=\beta_{Q}\left[u u^{\prime}-\widehat{Q}\right]$, such that $\widehat{Q} \approx Q=$ $\left\langle u u^{\prime}\right\rangle$. For a given input presentation, these lateral connections evolve an auxiliary vector $v$ according to $v_{t}=v_{t-1}+u-$ $\alpha \widehat{Q} v_{t-1}$. Regardless of initial $v$, and assuming the scalar $\alpha$ is chosen so that $v$ converges, the Infomax anti-redundancy term can be approximated by iterating the lateral network 
TABLE 1 | Component models and parameters.

\begin{tabular}{|c|c|c|c|}
\hline Component & References & $\begin{array}{l}\text { Parameter } \\
\text { name }\end{array}$ & $\begin{array}{l}\text { Parameter } \\
\text { value }\end{array}$ \\
\hline \multirow{4}{*}{$\begin{array}{l}\text { Neocortex, layers } 4 \\
\text { and } 2 / 3\end{array}$} & Bell and Sejnowski, 1995; & $\beta_{x_{0}}$ & 0.00002 \\
\hline & Linsker, 1997; & $\beta_{w_{0}}$ & 0.0007 \\
\hline & Kozloski et al., 2007 & $\beta_{C}$ & 0.0007 \\
\hline & & $\beta_{Q}$ & 0.0007 \\
\hline \multirow[t]{9}{*}{ Neocortex, layer 5} & Zheng and Triesch, 2014 & $\eta_{\mathbb{I P}}$ & 0.01 \\
\hline & & $T^{E} \max$ & 1.0 \\
\hline & & $T^{\prime} \max$ & 0.5 \\
\hline & & $\mu_{\mathrm{IP}}$ & 0.1 \\
\hline & & $\sigma_{\mathrm{HIP}}$ & 0 \\
\hline & & $\eta_{\text {inhib }}$ & 0.001 \\
\hline & & $\sigma_{\xi}^{2}$ & 0.01 \\
\hline & & $\eta_{\mathrm{STDP}}$ & 0.004 \\
\hline & & $\eta_{\text {iSTDP }}$ & 11.0 \\
\hline \multirow[t]{10}{*}{ Striatum } & Rabinovich et al., 2001 & $g \max$ & 0.25 \\
\hline & & $g_{\min }$ & 0 \\
\hline & & a & 0.7 \\
\hline & & $b$ & 0.8 \\
\hline & & $\tau_{1}$ & 0.08 \\
\hline & & $\tau_{2}$ & 4.1 \\
\hline & & $v$ & -1.5 \\
\hline & & $x_{0}$ & -1.2 \\
\hline & & $y_{0}$ & -1.62 \\
\hline & & $z_{0}$ & 0 \\
\hline \multirow[t]{13}{*}{ Dopamine neurons } & Mihalas and Niebur, 2009 & $b$ & 1.0 \\
\hline & & $G / C$ & 50 \\
\hline & & $k_{1}$ & 200 \\
\hline & & $k_{2}$ & 20 \\
\hline & & $\Theta_{\text {inf }}$ & -0.05 \\
\hline & & $R_{1}$ & 0 \\
\hline & & $R_{2}$ & 1.0 \\
\hline & & $E_{L}$ & -0.07 \\
\hline & & $V_{R}$ & -0.07 \\
\hline & & $\Theta_{r}$ & -0.06 \\
\hline & & $a$ & 1.0 \\
\hline & & $A_{1}$ & 5.0 \\
\hline & & $A_{2}$ & -0.3 \\
\hline
\end{tabular}

and applying its output by Hebbian learning, since $\left(C^{\prime}\right)^{-1}=$ $Q^{-1} C\left\langle x_{I} x_{I}^{\prime}\right\rangle$, and by substitution, $\left(C^{\prime}\right)^{-1}=\alpha\left\langle v_{\infty} x_{I}{ }^{\prime}\right\rangle$ (Linsker, 1997).

- Neocortex, layer 5: The model evolves from a self-organizing recurrent network (SORN) of binary spiking units through application of homeostatic plasticity, weight normalization, and STDP learning rules, together with synaptic pruning and synaptogenesis (Zheng and Triesch, 2014). This biologically consistent set of synaptic modifications creates distributions of synaptic densities and weights that evolve over time to closely match data from developing neocortex. The weight matrix $W$ also develops robust feed forward motifs and synfire activity similar to the model of Kozloski and Cecchi (2010), but with the remarkable topological feature of a closed, global loop of distinct propagation layers (Figure 5), which together engender "synfire rings." We evolved this network for 200, 000 time steps $(\Delta t=1 \mathrm{~ms})$ to create four areas of cortex, which were then embedded into the larger model as two frontal lobe $\left(M_{1}, M_{\text {sup }}\right)$ and two sensory lobe $\left(S_{1}, S_{\text {sec }}\right)$ areas. Weights close to zero were held at zero for the remainder of all simulations. Propagating activity is maintained in the excitatory network, satisfying the requirement for layer 5 traversals. An inhibitory network that undergoes biologically plausible inhibitory STDP at its synapses onto excitatory neurons, together with homeostatic plasticity in the excitatory network, maintains spiking activity, $s(t)$, in the synfire ring at a nominal firing rate of 100 spikes/s. The inhibitory network imposes global, persistent competition across the network of excitatory layers. We propose this inhibition as an approximate functional model of inhibition from the thalamic reticular nucleus, which also integrates activity from across the thalamocortical system.

- Striatum: The model creates activation paths within the state space of a weakly connected, asymmetric inhibitory network to give rise to "winnerless competition" (Rabinovich et al., 2001), and alternating bouts of activity (i.e., "turn-taking") among the different neurons in the network (Figure 6). These bouts have been used by others to model the intrinsic dynamics of the striatum (Ponzi and Wickens, 2010), and together represent global attractor states that encode the modulatory and reorganizing influence of excitatory inputs to the network from cortical layer 5. Using a FitzHugh-Nagumo model, MSNs are represented by three dynamic variables. First, $x_{f}(t)$ in the model represents the "burst potential" of the neuron, with a positive transient in this potential representing a $\sim 350 \mathrm{~ms}$ burst. Computed using the same time step as the binary spiking layer 5 model, this coarse resolution model of the neuron's membrane potential is appropriate given the dominant bursting mode of firing in MSNs, and the observation that activity is often observed as alternating series of bursts of bursts (Miller et al., 2008). The remaining variables $y_{f}(t)$ represent a recovery from inhibition and $z_{f}(t)$ the inhibitory synaptic current received by the neuron, summed over the inhibitory inputs from other neurons through a Heaviside step function and the inhibitory weights $W_{\text {Str }}$.

- Dopamine Neurons: The model is that of a leaky integrate and fire (IAF) neuron. Four state variables are computed: a membrane potential $V(t)$, a variable threshold $\Theta(t)$, and two intrinsic currents $I_{1}(t)$ and $I_{2}(t)$, each integrated over the same time step as the previous two models. Because spikes in this model are represented by instantaneous resets of each variable at $V(t)>\Theta(t)$, the time step $(\Delta t=1 \mathrm{~ms})$ is sufficient to integrate the neuron's spiking dynamics. Based on the published model, we derived an instance of a "rebound burst" model, and satisfied the requirements of dopamine neurons in the closed striato-nigro-striatal loop. Specifically, 

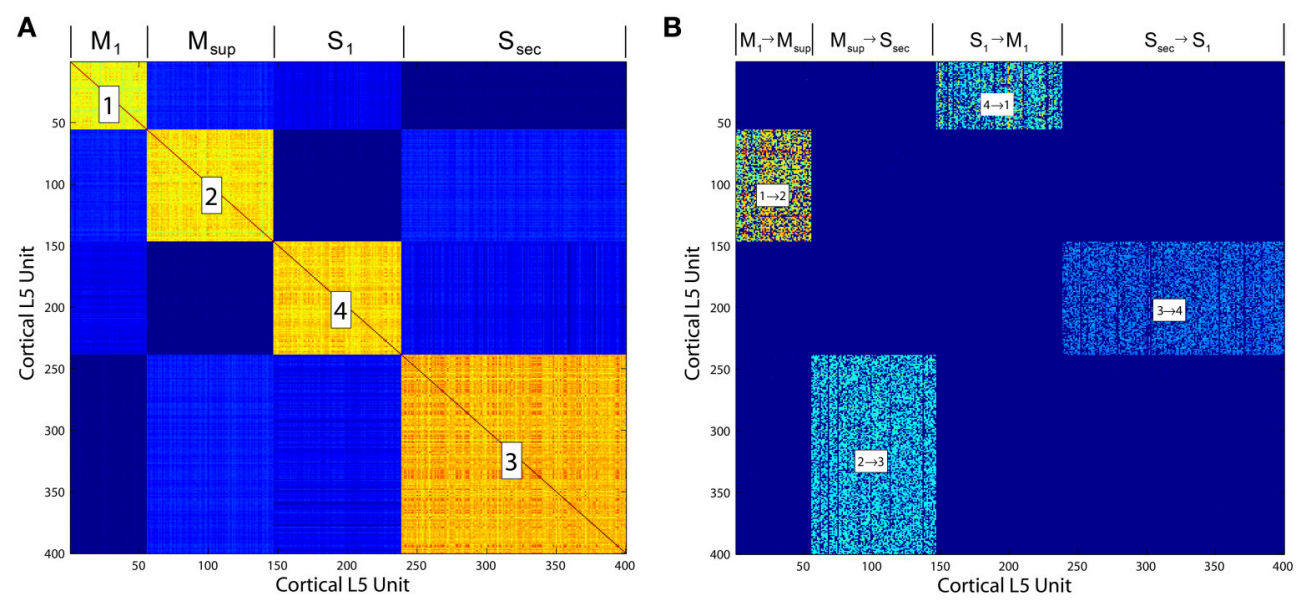

FIGURE 5 | (A) Correlation matrix computed over the final 10, 000 iterations of a simulation of the Layer 5 model based on Zheng and Triesch (2014). The four self-organized layers of this cortico-cortical topology are correlated in firing. (B) The cortico-cortical feedback weight matrix, showing clear dominance of the feed forward area to area connections over all others. This self-organized topology supports synfire ring activity, which in the current model is referred to as traversal activity.

the voltage-dependence of $\Theta(t)$ permits the model to generate rebound spiking under conditions when the neuron has been hyperpolarized deeply, or for a prolonged period (Figure 7). Due to the independent spike-induced current $R_{2}$, each rebound event generates a burst of four action potentials. This simplification's phenomenology also approximates that generated by other more complex models of rebound firing in dopamine neurons (Lobb et al., 2011).

\subsection{Component Model Interfaces}

The interfaces between component models that implement the integrated brain model of information-based exchange summarized in Algorithm 1, are now listed and described.

- Feedback Cortico-cortical: Layer 5 feedback inputs to a cortical area layer 5 are modeled as in the self-organizing recurrent network of Zheng and Triesch (2014) and implement the traversal network. Inputs are also categorized by the layer $2 / 3$ model as an input vector of sums of binary spike trains over a time window $\tau_{X}$. This vector $\widehat{x}_{\mathrm{FB}}(t)$ comprises the elements $\widehat{x}_{\mathrm{FB}_{i}}(t) \leftarrow \sum_{t-\tau_{X}}^{t} s_{\mathrm{FB}_{i}}(T)$, where $s_{\mathrm{FB}_{i}}(T) \in\{0,1\}$ is the spike train from one unit in the upstream layer 5 area. Because of the full rank requirement of the information maximizing algorithm, the model of layer $2 / 3$ includes a fixed first stage random mixing matrix $M_{\mathrm{FB}}$, drawn from a lognormal distribution with unit mean and unit standard deviation, and linearly combining the elements of $\widehat{x}_{\mathrm{FB}}$ to create the feedback input vector $x_{\mathrm{FB}} \leftarrow M_{\mathrm{FB}} \cdot \widehat{x}_{\mathrm{FB}}$.

- Feed Forward Cortico-thalamo-cortical: Inputs to a thalamic relay neuron $j$ projecting to a cortical area are modeled as a vector of sums over a time window $\tau_{X}$ of binary spike trains from layer 5 units in the cortical area projecting in the feed forward direction to the same area. This vector $\widehat{x}_{\mathrm{FF}}(t)$ then comprises elements $\widehat{x}_{\mathrm{FF}_{i}}(t) \leftarrow \sum_{t-\tau_{X}}^{t} s_{\mathrm{FF}_{i}}(T)$, and is similarly transformed by a mixing matrix such that the thalamic relay neuron's activity $\theta_{\mathrm{FF}} \leftarrow M_{\mathrm{FF}} \cdot \widehat{x}_{\mathrm{FF}}$. Each element $\theta_{\mathrm{FF}_{j}}$ is then subjected to the forward driver gating function $G(\cdot)$ over the gating vector $\widehat{G}$, such that elements of the feed forward input vector are $x_{\mathrm{FF}_{j}} \leftarrow \widehat{G}_{j} \cdot \theta_{\mathrm{FF}_{j}}$.

- Layer 4 Thalamic and Feedback Inputs : Feed forward thalamic inputs to layer 4 are combined with feedback inputs, such that the input vector to information maximization in layer $2 / 3, \widehat{x}_{I} \leftarrow x_{\mathrm{FB}}+x_{\mathrm{FF}}$. It is at this stage also that sensory inputs from a simulated environment may be added to the model.

- Layer 2/3 to Layer 5: The Layer 2/3 output vector $y$ provides an input to a gain function for layer 5 's integration of binary spikes from feedback traversals of the Grand Loop. This gain function is a model of the layer 5 neuron's apical dendrite, and is parameterized by the term $A c h \in[0,1]$, a proxy for the level of cholinergic modulation in neocortex. The gain on inputs to layer 5 unit $j$ is then $U_{j}=\left[1-\operatorname{Ach}\left(1-y_{j}\right)\right] /[1-A \operatorname{ch} / 2]$, which at $A c h=0$, preserves unitary gain regardless of $y$, and at $A c h=1$ provides a gain $U \in(0,2)$ for $y \in(0,1)$. In this way, assuming information maximization divides the population into different halves of active and inactive units, the total synaptic input to the network will remain constant, since the $U_{j}$ will always have a mean of 1 , and is applied multiplicatively to the excitatory synaptic integration function of each layer 5 neuron as in Zheng and Triesch (2014).

- Globus Pallidus to Thalamus: The forward driver gating function $G$ applied to thalamic relay neurons in the feed forward cortico-thalamo-cortical pathway models the final output of basal ganglia, a transient increase (via the indirect pathway) or decrease (via the direct pathway) in inhibition. $\widehat{G}$ is computed using a modified pallido-thalamic adjacency matrix $D$, comprising 1 for all direct pathway pallido-thalamic inputs, -1 for all indirect pathway pallido-thalamic inputs, and 0 for all unconnected pallidal to thalamic relay neurons. The bursting outputs of MSNs are represented by the half wave rectification function $P$, of the burst potential variable $x_{f}(t)$, and the gating vector is then $\widehat{G}=\mathcal{H}\left[D \cdot P\left(x_{f}(t)\right)\right]$, where $\mathcal{H}$ is the Heaviside function. 
A
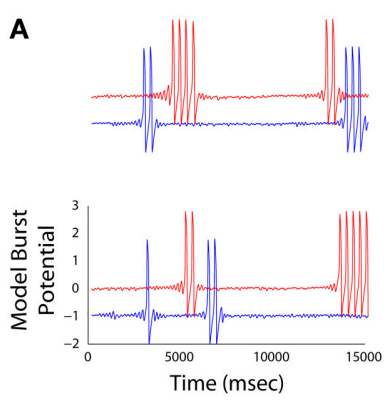

B
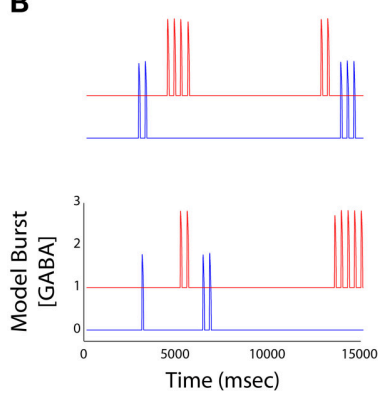

C
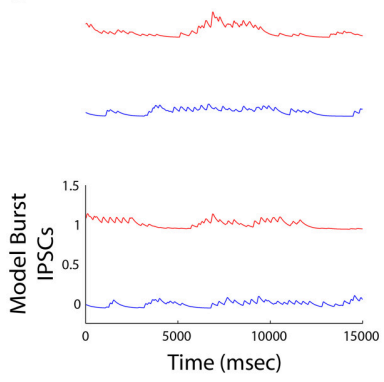
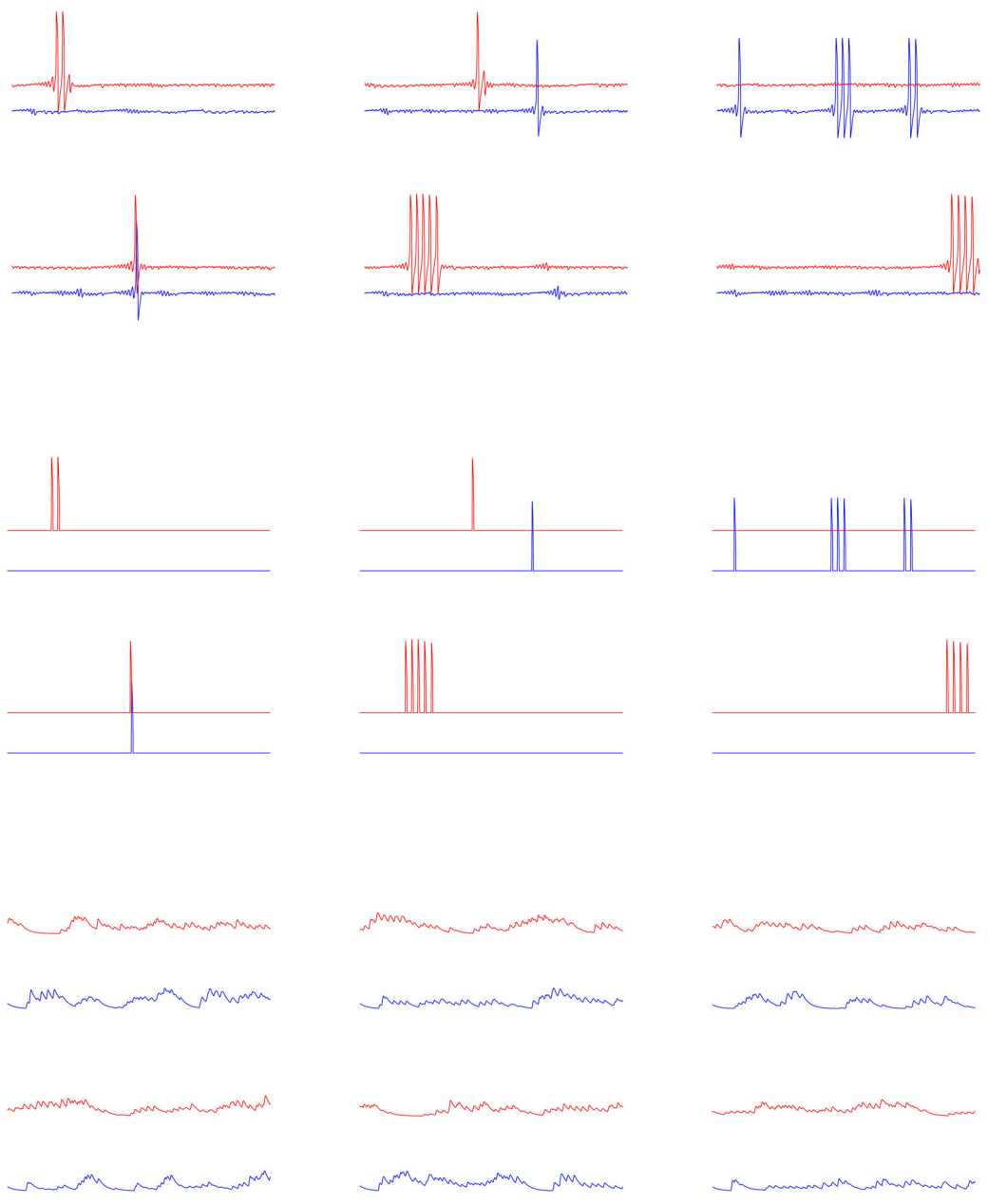

$-$

FIGURE 6 | (A) Striatal burst potential time series derived from FitzHugh-Nagumo models of four MSNs (columns) plotted at the beginning (blue) and the end (red) of a simulation. Each burst potential represents a series of MSN spikes fired in a burst. (B) The time aligned burst output traces represent the half wave rectified version of the potentials in (A). (C) IPSCs received by each MSN (outward currents plotted as a positive deflection). Currents are maximal when the neurons bursting ceases, and are low when it is bursting, reflecting the operation of winnerless competition in the lateral inhibitory network.

- Layer 5 to Striatum: The inputs from the Layer 5 model to an MSN in the Striatum model are drawn from all layer 5 neurons in the cortical area for which the MSN gates inputs at the thalamus, and from those in the areas connected to it in either the feed forward or feedback directions. These Layer 5 inputs may also be directed to motor outputs of the model to a simulated environment (as in the Pyramidal tract). Corticostriatal synapses are subjected to STDP that differentially adjusts weights, $W_{\mathrm{CStr}}$, based on correlation between cortical spiking and the derivative of the burst outputs of MSNs, $P^{\prime}\left(x_{f}(t)\right)$. Pre-post pairing is defined as when a cortical spike occurs and this derivative is positive, and postpre pairing when a cortical spike occurs and it is negative. Each kind of pairing is computed separately and subjected to the modulatory conditions at the synapse, as illustrated in Figure 4. Briefly, depending on 1. the identity of the MSN (D1- or D2-type), 2. whether dopamine is or is not present at the synapse, and 3. whether the inhibitory synaptic current $z_{f}(t)$ at the MSN exceeds a threshold $\left(z_{f}(t)>0.00707\right)$, each pairing value may be either 1 or 0 , and the adjustment to the weight a multiple of this value and a learning rate of 0.002 . As in Zheng and Triesch (2014), weights are normalized such that the sum of all inputs to an MSN cannot exceed 0.1 . When weights reach zero they are pruned, and a single new connection may then be formed during a time step with probability 0.2 .

- Striatum to Dopamine Neurons: The input to each dopamine neuron in the model, $I_{e}$ (Mihalas and Niebur, 2009), is computed by summing all burst outputs from those MSNs projecting to the dopamine neuron, multiplied by a constant weight of -2.25 .

- Dopamine Release to Corticostriatal Synapses: Unlike all other projections in the model's interfaces, the dopamine 

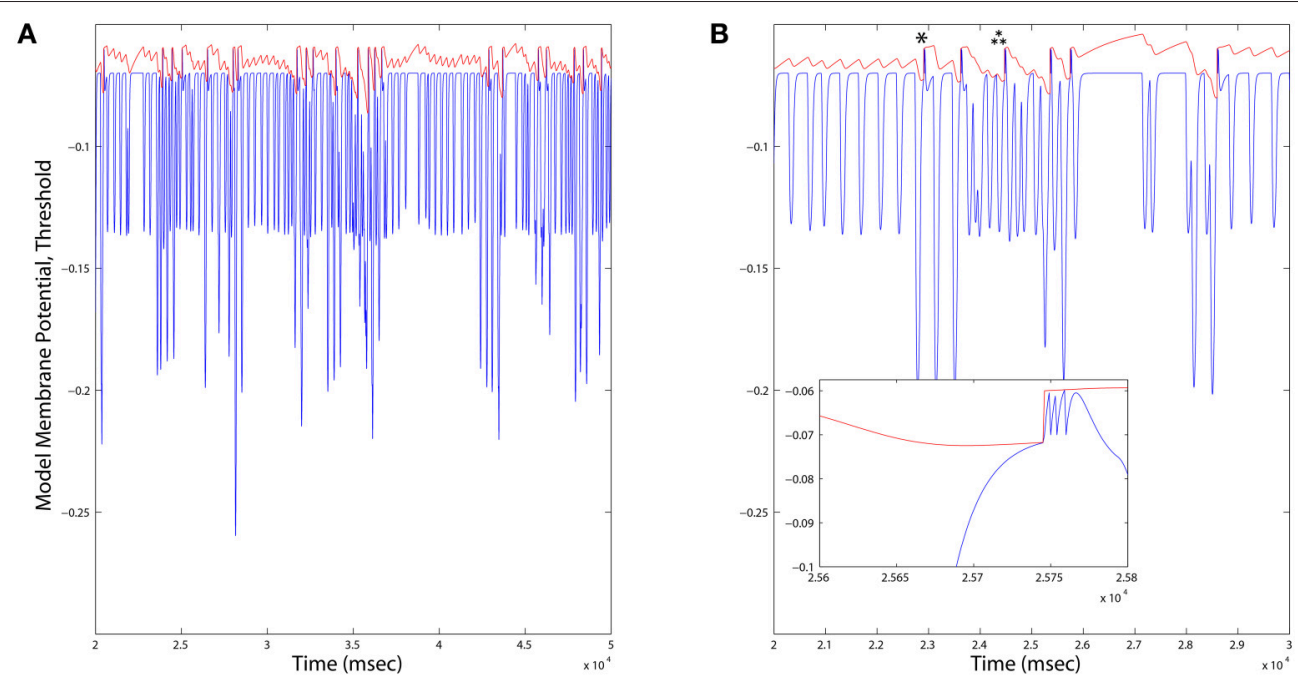

FIGURE 7 | (A) Long duration time series plots of Dopamine Neuron model variables. When the membrane potential (blue) reaches the variable threshold (red), a spike reset occurs. Firing rates of Dopamine neurons across all simulations were consistently on average $\sim 1.6 \mathrm{~Hz}$, and varied locally depending up the ongoing integration of dynamic inputs. (B) An expanded time scale reveals bursts (inset) occurring in response to deep hyperpolarization (single star), or prolonged weaker hyperpolarization (triple star) events.

neuron projection is to a synapse, not a neuron. Specifically, dopamine spiking results in a persistent dopamine modulation of STDP at a specific set of corticostriatal synapses. Dopamine neurons are assigned randomly without replacement to corticostriatal synapses onto each MSN. The duration of dopamine modulation following a Dopamine Neuron model spike persists at the synapse for a time $\tau_{\mathrm{DA}}$.

\subsection{Simulation Materials and Experiments}

We simulated the model to explore the rate and heterogeneity of transitions in traversals and in subcortical modulators of these traversals. The configuration (Table 2) allowed for a rapid prototyping in Matlab because of the simulation's small size. Following initialization of the cortico-cortical Grand Loop network of four areas, we simulated the full model for an additional 500, 000 iterations using an Intel Xeon E5-2640 v3 Processor (20 MB Cache, $2.60 \mathrm{GHz}$ ), requiring 2.5 hours of compute time. The first 50, 000 iterations were used to adjust the biases of the layer 2/3 model, during which time Ach modulation of layer 5 was drawn from the positive half of a zero mean normal distribution with standard deviation of 1 . All plots, except where noted, show the final iterations of the 500, 000 total. Reported are experiments wherein the parameter $\tau_{\mathrm{DA}}$ was set at either 25 or $100 \mathrm{~ms}$, and Ach at $0.25,0.5$, or 0.75 . All plots except Figure 12 show results for $\tau_{\mathrm{DA}}=100 \mathrm{~ms}$.

\section{SIMULATION RESULTS}

\subsection{Coordinated Behavior Among Component Models}

Behavior of the model may be analyzed first based on inspection of various raster plots from different components of the model.

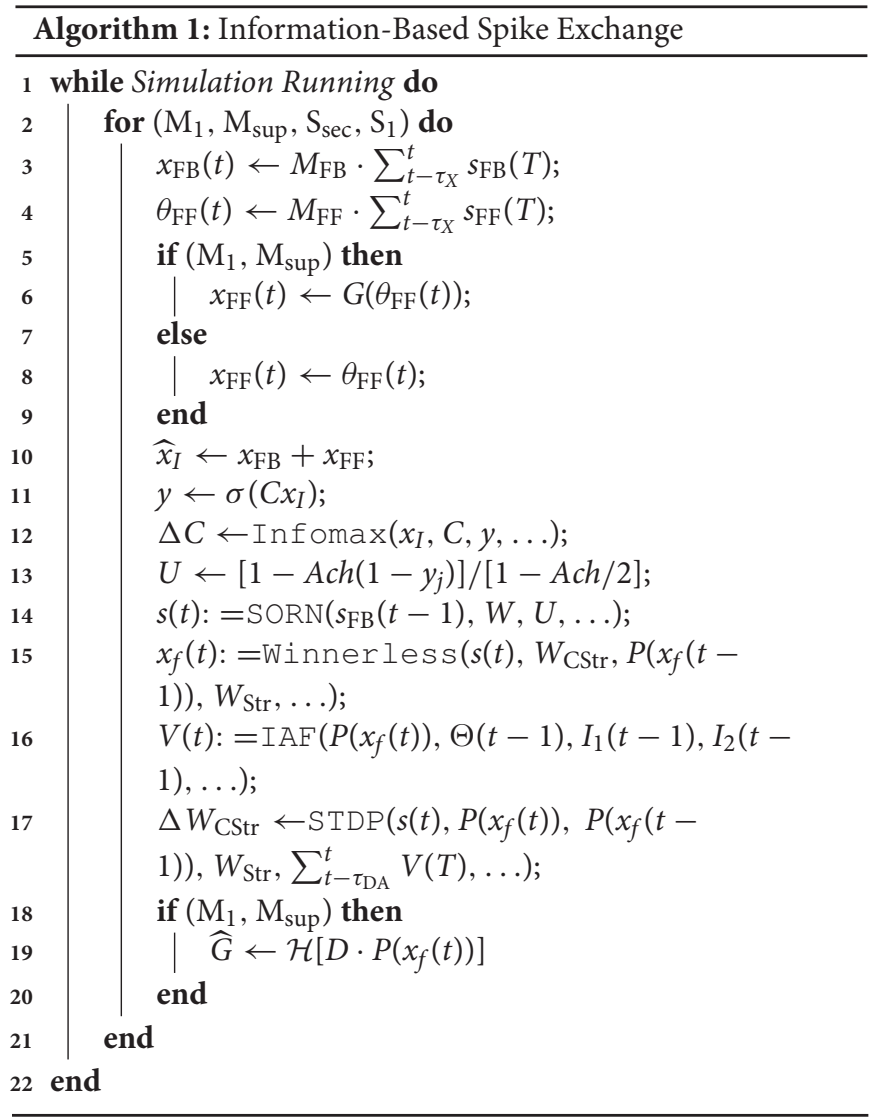

In this way coordination between the different components is apparent. We first observed that cortico-cortical traversals through the feedback layer 5 network occur without subcortical 
TABLE 2 | Configuration parameters specific to model simulation.

\begin{tabular}{|c|c|c|}
\hline $\begin{array}{l}\text { Parameter } \\
\text { name }\end{array}$ & Description & Parameter value \\
\hline$N_{A}$ & Number of cortical areas & 4 \\
\hline$N_{E}$ & $\begin{array}{l}\text { Number of thalamocortical units (layer } 5 \text {, } \\
2 / 3 \text { pyramidal, thalamic relay neurons) }\end{array}$ & 400 \\
\hline$\tau_{X}$ & $\begin{array}{l}\text { Layer } 2 / 3 \text { integration window for spiking } \\
\text { to rate code transformation (ms) }\end{array}$ & 100 \\
\hline$N_{l}$ & $\begin{array}{l}\text { Number of thalamic reticular inhibitory } \\
\text { neurons }\end{array}$ & 80 \\
\hline$N_{\text {Frontal }}$ & $\begin{array}{l}\text { Number of frontal cortical areas under } \\
\text { striatal gating }\end{array}$ & 2 \\
\hline$N_{\text {Str }}$ & Number of striatal MSNs & 100 \\
\hline$N_{\mathrm{CX}, \mathrm{Str}}$ & $\begin{array}{l}\text { Number of cortical neurons projecting to a } \\
\text { striatal neuron }\end{array}$ & 20 \\
\hline$W_{\mathrm{C}_{\mathrm{C}, \mathrm{Str}}}$ & Initial corticostriatal weight & $0.005,\left(0.1 / N_{\mathrm{Cx}, \mathrm{Str}}\right)$ \\
\hline$N_{\text {Str, Th }}$ & $\begin{array}{l}\text { Number of striatal neurons projecting to a } \\
\text { thalamic relay neuron }\end{array}$ & 11 \\
\hline$N_{\mathrm{DA}}$ & Number of dopamine neurons & 20 \\
\hline$N_{\text {Str,DA }}$ & $\begin{array}{l}\text { Number of striatal neurons projecting to a } \\
\text { dopamine neuron }\end{array}$ & 20 \\
\hline$\tau_{\mathrm{DA}}$ & Dopamine modulation window (ms) & 25,100 \\
\hline
\end{tabular}

regulation, and were similar to the synfire events reported by Zheng and Triesch (2014). There are two main regulators of these traversals in our model: (1) an information based gain on layer 5 feedback inputs provided from layer $2 / 3$, and (2) basal ganglia gating of cortico-thalamo-cortical feed forward inputs to layer $2 / 3$ information maximization by the forward driver gate.

Upon introducing these regulators, we noted that traversals became structured into long bouts of smoothly alternating and repeating patterns of activity across the different cortical layers' raster plots. Each pattern persisted for $\sim 400 \mathrm{~ms}$ (Figure 8A), and sequences of patterns, while similar over each cycle, were not identical. The Ach parameter provides a means to adjust the influence of categories learned by layer $2 / 3$ on traversals. For this initial experiment, $A c h=0.25$ provided a gain $U \in(0.86,1.14)$ for $y \in(0,1)$.

Information maximization creates maximal entropy in the ensemble of output vectors over an input ensemble, and because of the logistic function, activity in each layer $2 / 3$ neuron was typically close to zero or one. We interpret these values as cortical up and down states, which have both an extrinsic and intrinsic origin in the local cortical microcircuit.

Maximizing entropy of the ensemble of gain functions, applied to layer 5 inputs in the feedback traversal network, had the interesting effect of creating more irregularity in the patterns of activity across all of cortex as Ach increased. At Ach $=0.5, U \in$ (0.67, 1.33), (Figure 8B), pattern combinations became varied, even though average global firing rates imposed by homeostatic plasticity in the network were consistently maintained (100 spiked/s). Finally, at $A c h=0.75, U \in(0.4,1.6)$, traversal transition rates increase significantly, and patterns were highly varied (Figure 8C).

Inspecting the information-bearing up and down states in layer $2 / 3$ directly in state rasters from all four cortical areas also reveals coordination between areas and with transitions in traversals. In Figure 9A, under Ach $=0.25$, the rate of state changes among layer $2 / 3$ units appeared coordinated, especially in the secondary sensory area. This coordination is less regularly transitioned than in the traversals, and occurs at a higher rate. At higher Ach $=0.5$ (Figure 9B) up and down state coordination with traversals increases, while coordination across layer $2 / 3$ is weakened. At Ach $=0.75$ (Figure 9C) states become synchronized in the secondary sensory area and more coordinated with traversals overall, even though traversals themselves become more heterogeneous. Note that the heterogeneity in traversals due to increased control by the information maximizing network is not due to a lack of convergence in the weights of the networks. Weights among both the layer $2 / 3$ Infomax input weights $C$ and layer 5 feedback weights $W$ converged during these simulations.

MSN bursts generated by the model were ongoing, as in the winnerless network and the model of Ponzi and Wickens (2010). These bursts appeared in fast sequences, which were of longer duration in D1-type MSNs than D2-type (Figure 10). Variability in burst rate between MSNs was also observed, with some not firing at all, likely because of inhibition from the active network. Increasing Ach had only a small effect on the raster appearance, and so we began our quantitative analysis by examining coordination between the Striatum model and the Layer 5 model.

\subsection{Measurements of Information-Based Exchange}

To quantify coordination between striatum bursting and cortical layer 5 spiking, we computed pairwise linear correlation coefficients between each cortical spike train and striatal burst train. We plotted each using a color scale (red, more correlated; blue, less correlated) in a matrix showing how different areas of cortex fired in relation to D1- and D2-type MSN bursts (Figure 11, left column). Only significant correlations were plotted, and all others were represented by zero. We also show that the mean of each distribution of correlation values (Figure 11, right column) for both D1- (blue) and D2-type (red) MSNs differed. Most coefficient distributions of D1 vs. D2 burst correlation with cortical spiking were significantly different $(p<$ $0.05)$, based on pairwise student $t$-tests. More striking is the difference in sign for each mean coefficient of correlation to each cortical area as Ach increases. Positive correlation coefficients dominated at low Ach and negative at high. At the intermediate level, M1 in particular showed a divergence in sign between mean correlation coefficients for D1-type (positive) and D2-type (negative) MSNs.

Finally, to quantify information-based exchange directly, we measured the entropy of cortical spiking and dopamine neuron spiking, and the mutual information between cortical and dopamine neuron spiking (Strong et al., 1998). Instead of measuring entropy and information among spike trains of individual neurons however, which quantifies the distribution of patterns of spikes over time, we measured entropy and information in population spiking, which 

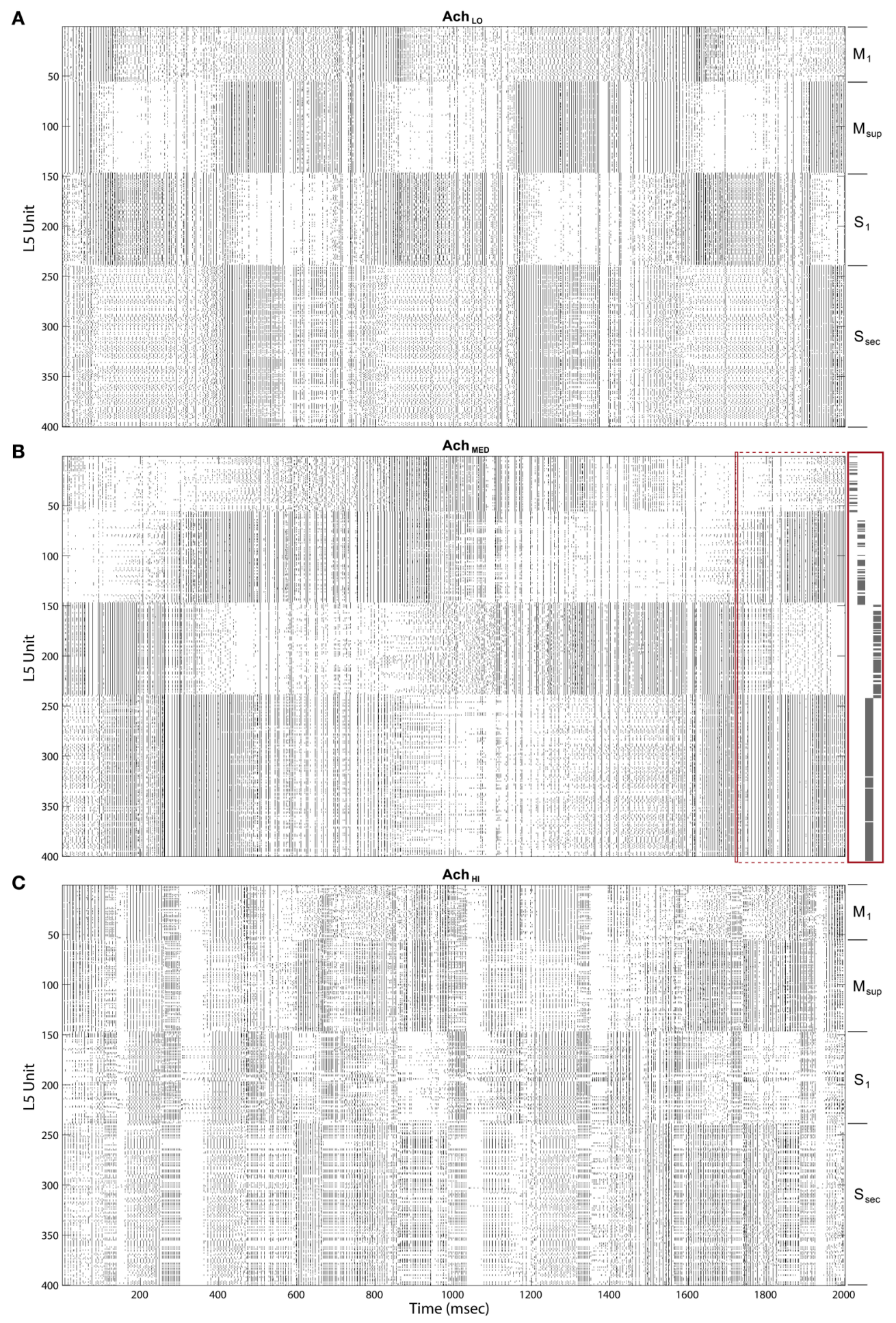

FIGURE 8 | Raster plots of the cortical Layer 5 model outputs. 400 spike trains from the final 2 s of simulated time. Cortical areas noted on right. (A) Under low Ach (0.25) patterns of traversals are long lasting ( 400 ms) and smooth. (B) Under moderate Ach (0.5) patterns of traversals become briefer and choppy, with example of a single traversal of the Grand Loop expanded on right (red boxes). (C) Under high Ach (0.75) patterns of traversals are brief (100-200 ms) and heterogeneous.

quantifies the distribution of patterns of spikes over the population for single time steps. The method was aimed at asking if traversals themselves show entropy maximization based on increased modulation from layer 2/3. Synfire events are encoded by the sets of units that participate at every stage of the chain or ring propagation. Therefore, 

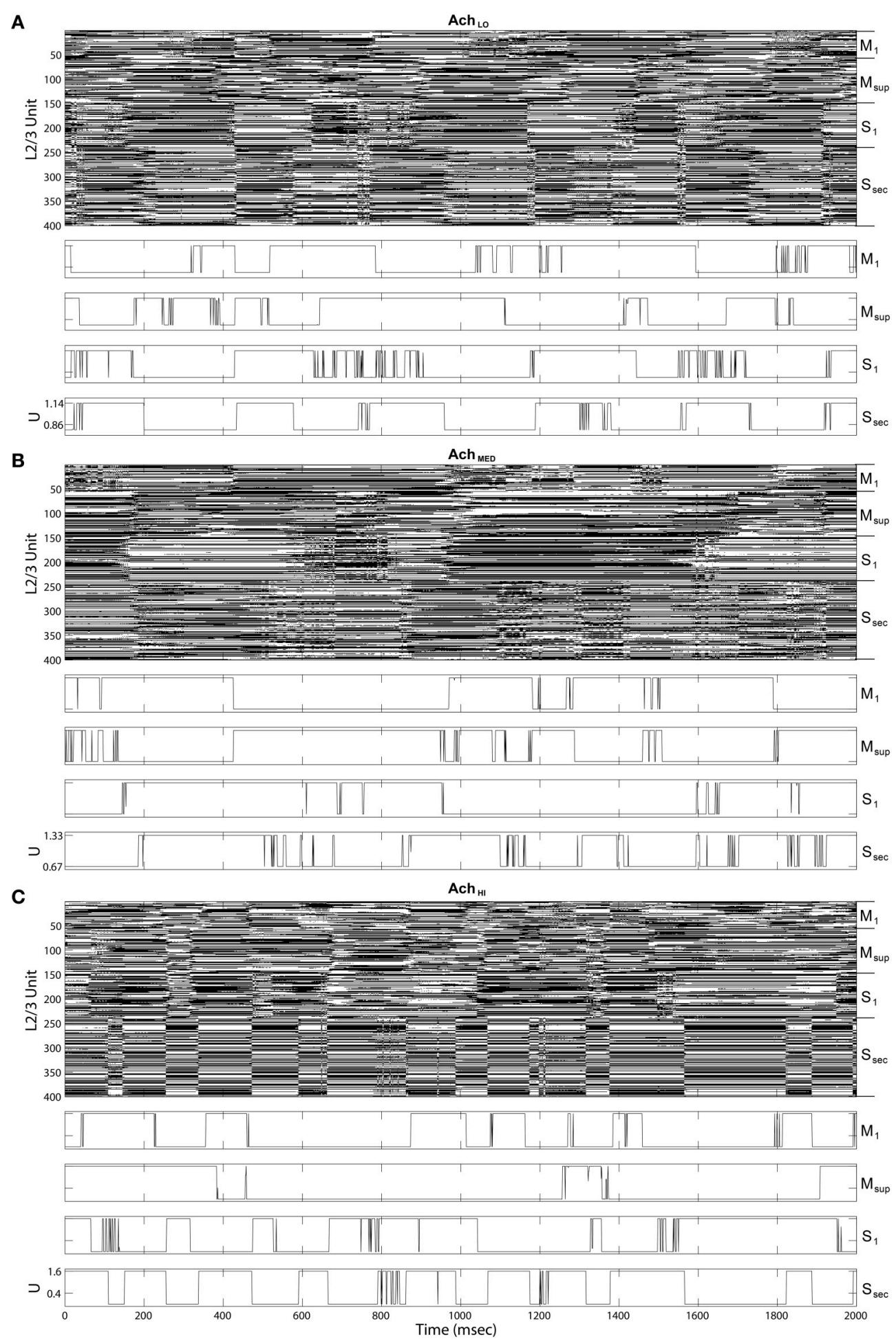

FIGURE 9 | Up state (black) raster plots of Layer 2/3 model outputs (upper panels) and example time series of gain $U$ on traversal inputs to each area (lower panels). (A) Under low Ach (0.25), states transition more quickly than traversals from Figure 8. (B) Under moderate Ach (0.5), states transition more slowly in sensory areas. (C) Under high Ach (0.75), up and down states become synchronized in the secondary sensory area.

if the entropy of synfire population spiking increases, it can be concluded that the synfire chain entropy itself has increased.
We found that entropy in cortical layer 5 population spiking increased as Ach increased (Figure 12A). We also show that as the window of dopamine integration $\tau_{\mathrm{DA}}$ increased, the 

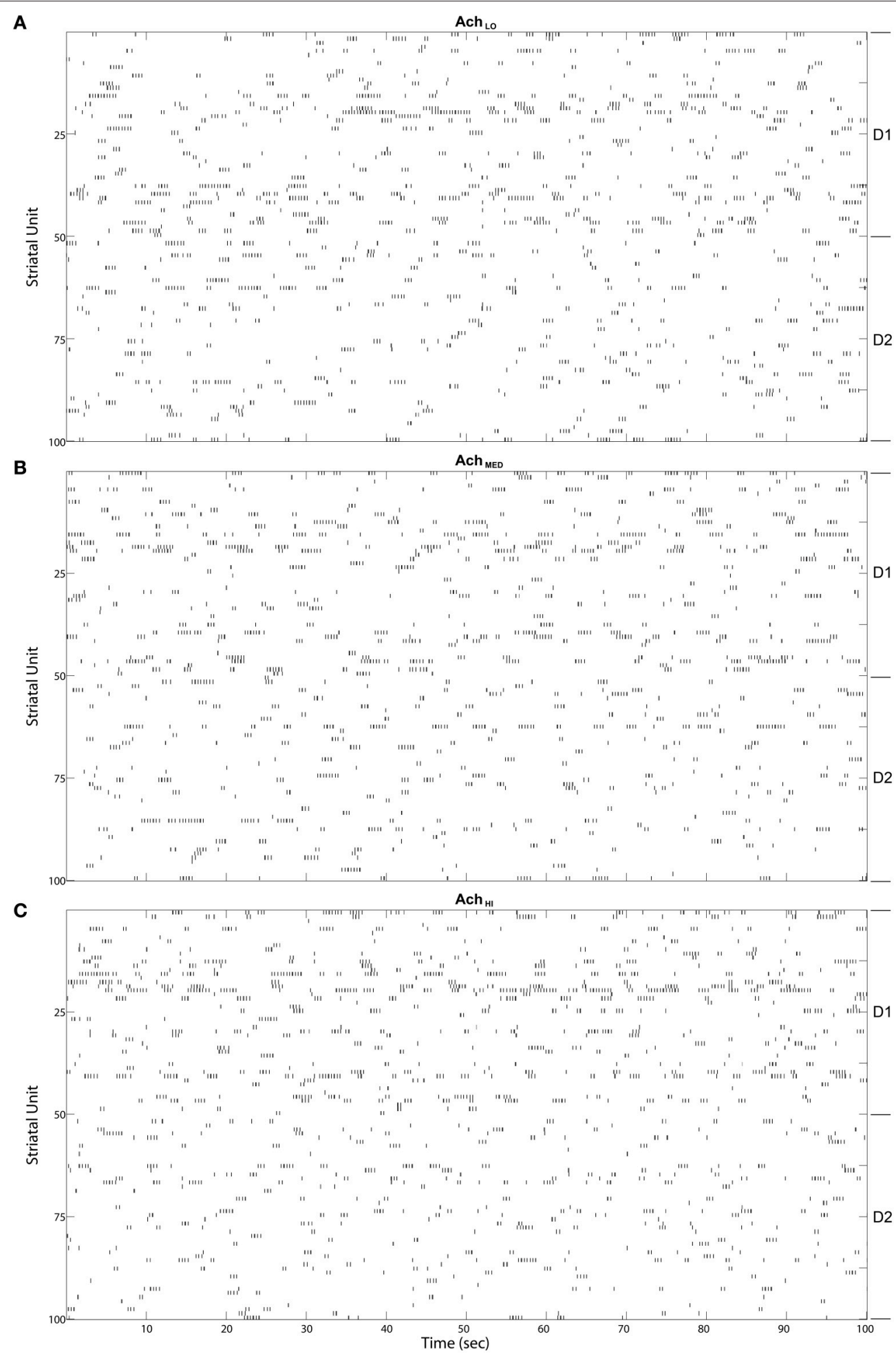

FIGURE 10 | Striatal model MSN burst raster plots over the final $100 \mathrm{~s}$ of simulated time. Bursts come in alternating bursts of bursts across the population due to winnerless competition. D1-type and D2-type of MSN noted at right. Bursts of bursts are longer in duration among D1-type MSNs under (A) Low (0.25), (B) Moderate (0.5), and (C) High (0.75) Ach. 

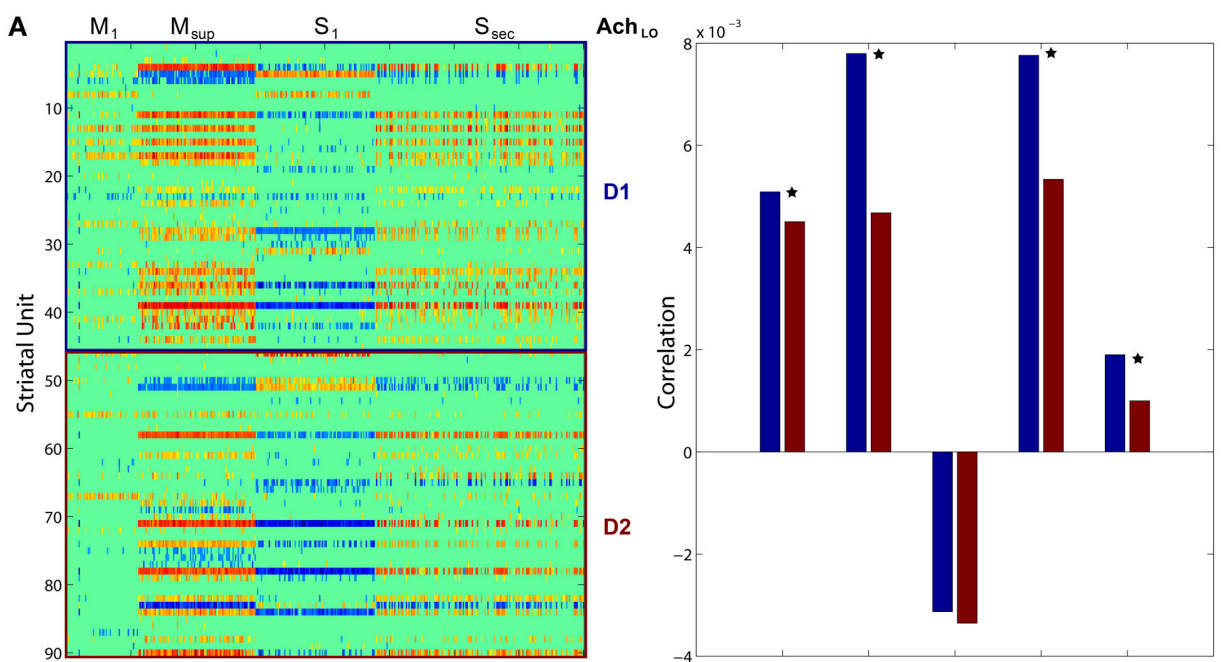

B

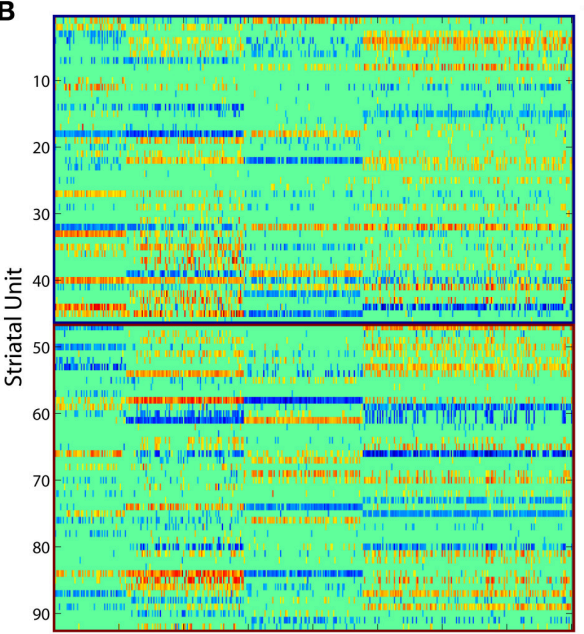

C

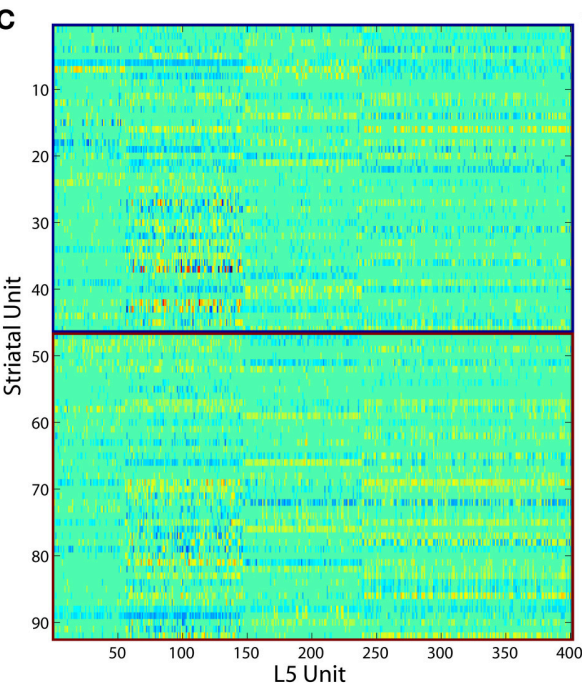

Ach $_{\text {MED }} \times 10^{-3}$

D1

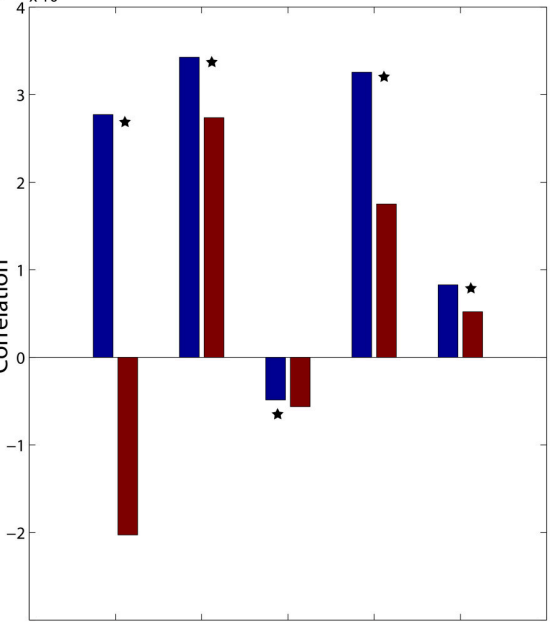

$\mathrm{Ach}_{\mathrm{HI}}$

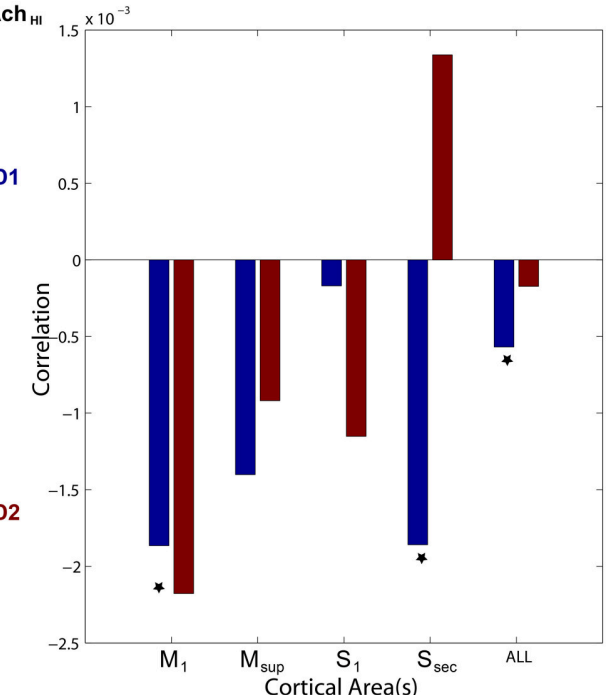

FIGURE 11 | Pairwise linear correlations coefficients between cortical spike trains and striatal bursts from Figures 8, 10. Only significant correlations are shown in the matrices plotted on left (D1- and D2-types noted along right) with others shown as zero. Mean correlation values are plotted on right, for D1- (blue) and D2-type MSNs (red). A star indicates significant difference $(p<0.05)$ in the distributions of coefficients (pairwise student $t$-test). (A) Under low Ach (0.25), correlations are positive across motor areas and all areas combined. (B) Under moderate Ach (0.5), correlations become positive for primary motor D1 and negative for D2. (C) Under high Ach (0.75), correlations are negative across motor areas and all areas combined. 


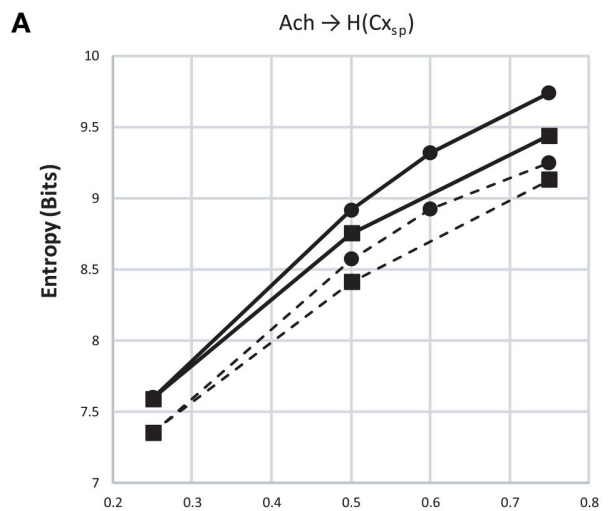

B

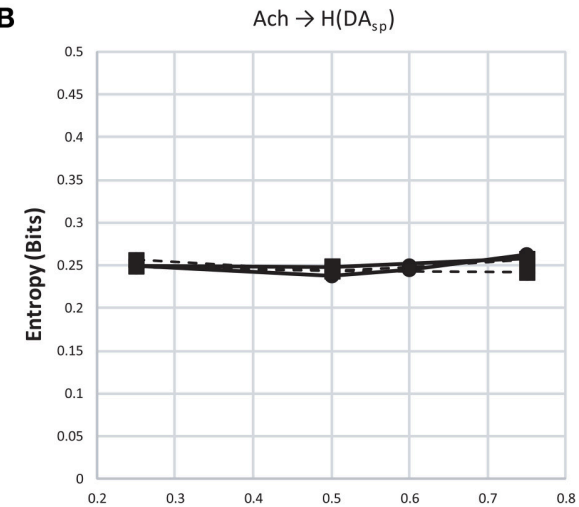

c

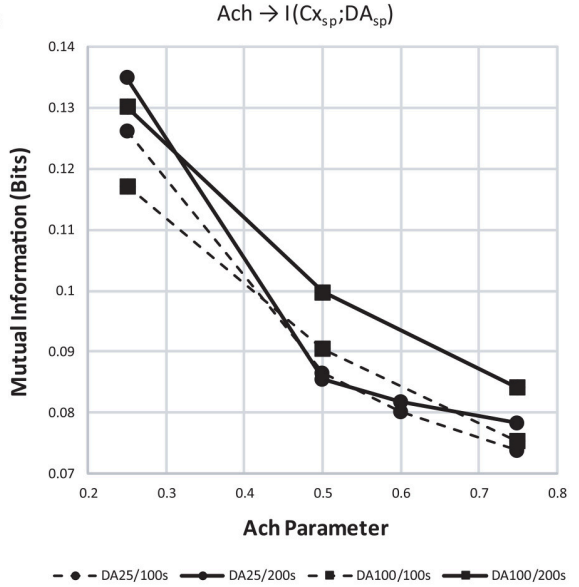

FIGURE 12 | Entropy and mutual information computed for cortical and dopamine neuron population spiking. (A) Entropy $(H)$ of cortical population spiking for final 100 (dashed) and 200 (solid line) seconds of simulated time under increasing Ach. Circles plot simulations with $\tau_{\mathrm{DA}}$ equal to $25 \mathrm{~ms}$ and squares $100 \mathrm{~ms}$. In all conditions $H$ increases with $A$ ch and $\tau_{D A}$. (B) Same as (A) but showing entropy of dopamine neuron population spiking. (C) With increasing cortical population spiking entropy (A), the mutual information between cortical and dopamine neuron population spiking decreased.

entropy of layer 5 population spiking increased slightly as well. Surprisingly, the entropy of dopamine neuron population spiking (Figure 12B) remained constant while both parameters in the model were altered. Finally, to measure how increasing traversal entropy depends on dopamine population spiking, we measured the mutual information between these two populations, and found it to decrease as Ach increased (Figure 12C).

\section{DISCUSSION}

We discuss the brain model of information-based exchange in three contexts: brain evolution and development, brain resting state networks, and new approaches to the study of brain disorders such as neurodegenerative diseases.

\subsection{Brain Evolution and Development}

We propose that the Grand Loop, spanning sensory, limbic, and motor cortices, and specifically traversing in our model somatosensory cortices, is prototypical and embryonic in origin, since other modalities develop fully only after birth and do not share a granular-agranular tiling boundary in von Economo's map. The topological relationship between other modalities and this backbone may then provide alternative pathways for completing a full traversal and rapidly binding percepts, needs, and behaviors. Finally, the tight coupling between somatosensory inputs and limbic states (i.e., tissue damage, pain) and motor states (i.e., sensorimotor feedback, proprioception) argues that this loop is likely preeminent in both brain evolution, organization, and development.

This model additionally provides insights into those organisms lacking cortices, wherein the stages of the proposed traversals may not be segregated anatomically (e.g., into Brodmann areas), but instead may be nucleated (e.g., in the birdsong system), or even superimposed within the same pallial regions (e.g., in fish and amphibians). Synfire ring development is robust given the synaptic modifications proposed by Zheng and Triesch (2014). It furthermore does not require anatomical segregation between layers to emerge or for synfire activity to propagate (e.g., for Figure 5, we sorted each matrix after areas developed in order to illustrate them clearly and connect subcortical structures to each).

Synfire rings may represent a prototypical substrate for behavior generation (Figure 13), and through subpallial regulatory inputs from thalamus and basal ganglia as described herein, for behavior selection. In such a scenerio, the evolution of a multilaminate neocortex to support such rings may have solved the problem of entropy maximization over the ensemble of synfire events in very large networks. Since the neural network implementation of Infomax requires a dense lateral network, to optimize each stage of a synfire ring and traversals in general would necessitate both the segregation of stages and a registered information maximizing network (Figure 13). This solution to the problem would then support rapid expansion of the synfire ring substrate by evolution, given that redundancy in large networks could suddenly be managed and eliminated by information maximization.

\subsection{Resting State Networks}

The challenge of modeling resting state activity in the brain has presented itself based on observations that distinct networks spanning multiple cortical areas appear in imaging studies to 


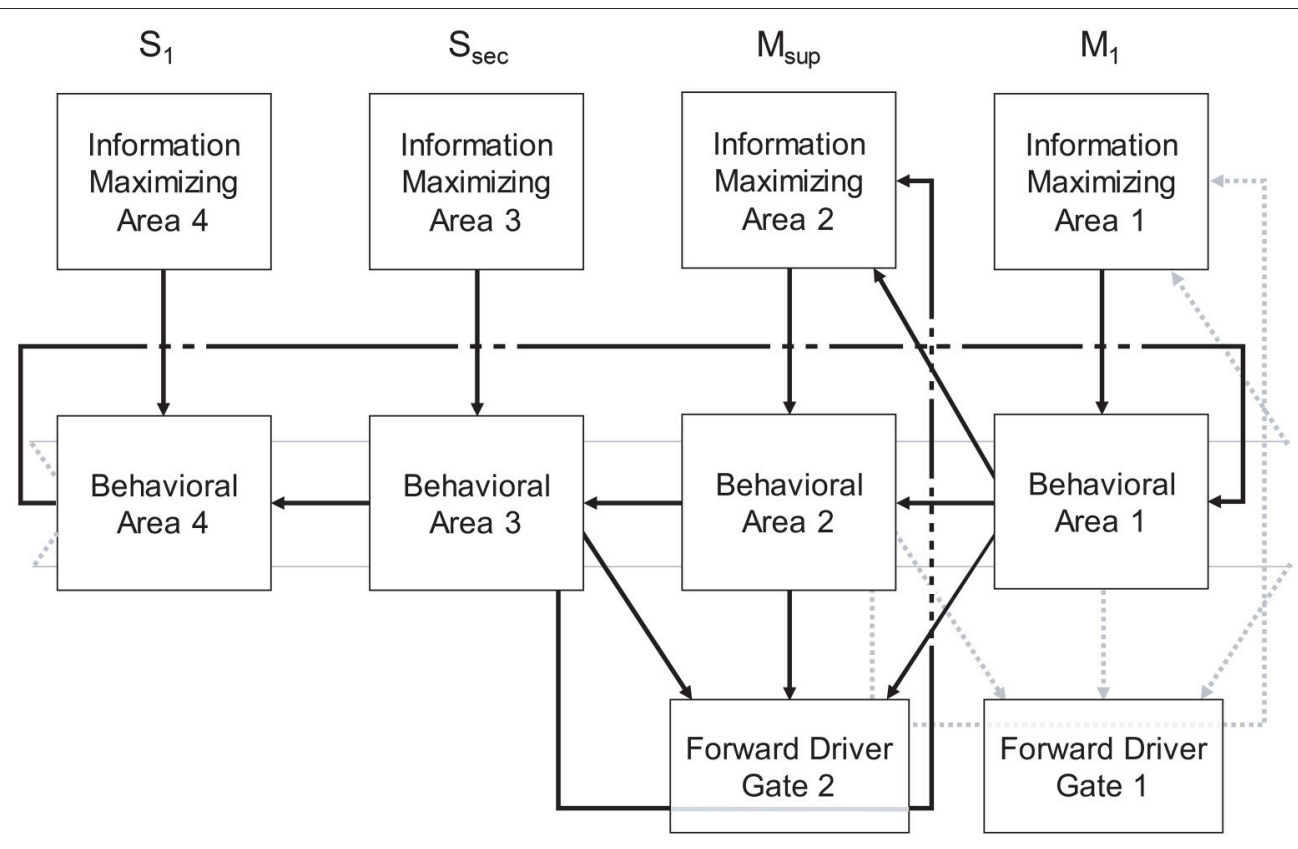

FIGURE 13 | A generalized schematic for an information-based exchange network.

serve either active or inactive states of the organism (Fox et al., 2005). Inactivity correlated networks appear even under anesthesia (Vincent et al., 2007), and these areas have very high metabolic rates, tipping the brain's energy budget toward a large investment in the organism's doing nothing.

What this costly outlay accomplishes may be explained by our model's use of closed-loop activity in the informationbased exchange network to increase entropy over the ensemble of traversals. In an evolutionary context, this activity may be viewed as preadapting the brain to selecting novel behaviors in novel contexts by maximizing such a quantity first, before engaging with the environment, then using the preadapted diverse traversals to explore it and seek reward.

While others have noted that resting state dynamics may represent a "constant state of inner exploration" (Deco et al., 2011), our model is the first to assign a quantitative measure to the fruits of this brain activity, providing a new way to reason about the trade-off between evolutionary pressures toward latent adaptive behaviors and the large metabolic cost of resting state network activity.

\subsection{Dynamic Disease Risk}

We hypothesize that basic controls are required to establish "cognitive homeostasis," i.e., a process by which variables that change brain dynamics are carefully regulated so that properties of brain state transitions (and thus brain information processing and behavioral dynamics) remain relatively stable under constant neuromodulatory conditions. We refer to these stable properties as "set points," i.e., targeted norms for critical system variables supporting normal behavior, percepts, affect, and cognition. In our model, these controls are based on a consistent set of parameters that yield consistent spiking and bursting patterns, even when the network undergoes reorganization (e.g., when Ach was modified, the system adjusted and produced stable traversals). Stable ranges of firing among burst rates and traversals, coefficients of correlated firing and bursts, and entropy and mutual information among population spiking and bursting have been our initial targets for describing these system set points using the brain model.

In real brains, given evolutionary pressures for robust selfregulation and behavior, the system is certainly replete with controls aimed at maintaining these set points. The challenge of studying brain disorders such as neurodegenerative disease is sorting primary and secondary risks from the multitude of compensatory mechanisms, each of which manifests itself as a deviation from normal brain and neuronal function given some primary genetic or injury risk. Researchers have shown, for example, that mutant Huntingtin protein disturbs NMDA receptor localization, densities, and currents at the corticostriatal synapse in mouse models of the disease (Cepeda et al., 2001). Knowing how this change arises and perturbs circuit dynamics, plasticity, and system set points may provide a better understanding of why certain neurons succumb and others don't when subjected to the same mutant protein.

We propose that perturbations in our model may result in stable dynamics, but with measurable risks related to stressors on normal neuronal function. If these deviations are extreme in our model, and therefore difficult to compensate for in biological tissue, a cascade of neuronal dysfunction may result. Neurodegenerative diseases such as Huntington's, Parkinson's, and Alzheimer's, may then be understood as cascading failures given initial stressors derived from plasticity abnormalities at the corticostriatal synapse, within the striato-nigro-striatal loop, and over the process of entropy maximization in layer $2 / 3$, 
respectively. For example, subtle changes to STDP or homeostatic plasticity may result in increased synaptic competition or cycling in the space of possible weights, which is then difficult to compensate for locally, given that traversals entail global brain states. If these risks increase when stressed neurons are removed from a simulation, the model may then be used to predict disease progression.

Implementation of the current brain model of informationbased exchange forms a framework for the analysis of cognitive homeostasis and disease using IBM's scalable approach to structural and neurophysiological modeling of neocortex and brain nuclei (Kozloski and Wagner, 2011). Here we extend this approach and that of many brain modeling projects, which seem focused on validating complex local circuit and tissue models at the expense of validating tissue inputs. Minimal complexity brain models, in our case an information-based exchange network, may be necessary to capture brain dynamics and provide validatable inputs to complex tissue models. The current model has now been reimplemented in the same model graph simulation infrastructure in which IBM's Neural Tissue Simulator was implemented, and thus will allow direct coupling between these in a single scalable, extensible program.

With this new approach, inputs and models of the various components may be simulated and compared to in vivo experimental observations. Furthermore, simulations over very long time scales can be used to stress the model and its set points in physiologically and clinically realistic ways. Additional perturbations to the model may include physiological stimulation, such as simulated deep brain stimulation (DBS) in simulated neural tissue, simulated drugs with known targets in the detailed model, and different simulated disease states with hypothesized mechanisms at the level of gene, protein, regulatory network, etc. Stimulation, drug effects, and disease mechanisms can then be targeted to test certain hypotheses about

\section{REFERENCES}

Abeles, M. (1991). Corticonics. Cambridge: Cambridge University Press. doi: 10.1017/CBO9780511574566

Amendola, J., Woodhouse, A., Martin-Eauclaire, M., and Goaillard, J. (2012). $\mathrm{Ca}^{2+} /$ cAMP-sensitive covariation of $\mathrm{I}(\mathrm{A})$ and $\mathrm{I}(\mathrm{H})$ voltage dependences tunes rebound firing in dopaminergic neurons. J. Neurosci. 32, 2166-2181. doi: 10.1523/JNEUROSCI.5297-11.2012

Bell, A. J., and Sejnowski, T. J. (1995). An information-maximization approach to blind separation and blind deconvolution. Neural Comput. 7, 1129-1159. doi: 10.1162/neco.1995.7.6.1129

Beul, S., and Hilgetag, C. (2015). Towards a "canonical" agranular cortical microcircuit. Front. Neuroanat. 8:165. doi: 10.3389/fnana.2014.00165

Binzegger, T., Douglas, R., and Martin, K. (2004). A quantitative map of the circuit of cat primary visual cortex. J. Neurosci. 24, 8441-8453. doi: 10.1523/JNEUROSCI.1400-04.2004

Cepeda, C., Ariano, M., Calvert, C., Flores-Hernndez, J., Chandler, S., Leavitt, B., et al. (2001). NMDA receptor function in mouse models of Huntington's disease. J. Neurosci. 66, 525-539. doi: 10.1002/jnr.1244

Deco, G., Jirsa, V., and McIntosh, A. (2011). Emerging concepts for the dynamical organization of resting-state activity in the brain. Nat. Rev. Neurosci. 12, 43-56. doi: $10.1038 / \mathrm{nrn} 2961$

Fino, E., Paille, V., Cui, Y., Morera-Herreras, T., Deniau, J.-M., and Venance, L. (2010). Distinct coincidence detectors govern the corticostriatal modifications to dynamic disease risk, and to study the wider system's behavior. Increasing complexity of perturbation sets (targets and combinations) may be designed to validate the model under different therapeutic conditions, and to test for phenotypic outcomes (e.g., symptomatology). Therefore, elaboration of these simulations within each modeled neural tissue might allow for in silico study of therapeutic interventions in living brain tissue.

In the above discussion, a model of several brain circuit components and their global set points has been proposed as a means to test disease mechanisms and therapeutic inputs such as DBS and drugs. The implicit assumption of these tests is that risks can be inferred from outlier variables that maintain system set points, and that these outliers may then be implicated as causes of phenotypic symptoms such as abnormal behavior at the organismal, circuit, neuronal, or synapse level. Targeting these variables in real world systems is one approach we propose for novel therapeutic design and discovery using brain modeling combined with neural tissue simulation.

\section{FUNDING}

A portion of this work was funded by CHDI.

\section{ACKNOWLEDGMENTS}

We acknowledge many years of helpful discussions with Charles C. Peck and Guillermo Cecchi. We also acknowledge discussions with Ralph Linsker and Roger Traub on component model design, and Robert Rogers and Robert Kerr on the integrated model design. We acknowledge helpful comments on the manuscript from Erik Schomburg, Tuan Hoang Trong, and Pengsheng Zheng. Finally, we acknowledge the artistic contributions of Stella Kozloski, who produced the drawings based on von Economo's work.

spike timing-dependent plasticity. J. Physiol. 588, 3045-3062. doi: 10.1113/jphysiol.2010.188466

Fox, M. D., Snyder, A. Z., Vincent, J. L., Corbetta, M., Van Essen, D. C., and Raichle, M. E. (2005). The human brain is intrinsically organized into dynamic, anticorrelated functional networks. Proc. Natl. Acad. Sci. U.S.A. 102, 96739678. doi: 10.1073/pnas.0504136102

Goldberg, J. H., Farries, M. A., and Fee, M. S. (2012). Integration of cortical and pallidal inputs in the basal ganglia-recipient thalamus of singing birds. $J$. Neurophysiol. 108, 1403-1429. doi: 10.1152/jn.00056.2012

Guillery, R. W., and Sherman, S. M. (2011). Branched thalamic afferents: what are the messages that they relay to the cortex. Brain Res. Rev. 66, 205-219. doi: 10.1016/j.brainresrev.2010.08.001

Hasselmo, M. E., and Giocomo, L. M. (2006). Cholinergic modulation of cortical function. J. Mol. Neurosci. 30, 1559-1166. doi: 10.1385/JMN:30:1:133

Hopfield, J. (1982). Neural networks and physical systems with emergent collective computational abilities. Proc. Natl. Acad. Sci. U.S.A. 79, 2554-2558. doi: 10.1073/pnas.79.8.2554

Kelly, R., and Strick, P. (2004). Macro-architecture of basal ganglia loops with the cerebral cortex: use of rabies virus to reveal multisynaptic circuits. Prog. Brain Res. 143, 449-459. doi: 10.1016/s0079-6123(03) 43042-2

Kozloski, J., and Cecchi, G. (2010). A theory of loop formation and elimination by spike timing-dependent plasticity. Front. Neural Circuits 4:7. doi: 10.3389/fncir.2010.00007 
Kozloski, J., Cecchi, G., Peck, C., and Rao, A. (2007). Topographic infomax in a neural multigrid. Lect. Notes Comput. Sci. 4492, 500-509. doi: 10.1007/978-3540-72393-6-60

Kozloski, J., and Wagner, J. (2011). An ultrascalable solution to large-scale neural tissue simulation. Front. Neuroinform. 5:15. doi: 10.3389/fninf.2011.00015

Lévesque, M., Gagnon, S., Parent, A., and Deschênes, M. (1996). Axonal arborizations of corticostriatal and corticothalamic fibers arising from the second somatosensory area in the rat. Cereb. Cortex 6, 759-770. doi: $10.1093 /$ cercor/6.6.759

Linsker, R. (1997). A local learning rule that enables information maximization for arbitrary input distributions. Neural Comput. 9, 1661-1665. doi: 10.1162/neco.1997.9.8.1661

Lobb, C., Troyer, T., Wilson, C., and Paladini, C. (2011). Disinhibition bursting of dopaminergic neurons. Front. Syst. Neurosci. 5:25. doi: $10.3389 /$ fnsys.2011.00025

Mihalas, S., and Niebur, E. (2009). A generalized linear integrate-and-fire neural model produces diverse spiking behaviors. Neural Comput. 21, 704-718. doi: 10.1162/neco.2008.12-07-680

Miller, B. R., Walker, A. G., Shah, A. S., Barton, S. J., and Rebec, G. V. (2008). Dysregulated information processing by medium spiny neurons in striatum of freely behaving mouse models of Huntington's disease. J. Neurophysiol. 100, 2205-2216. doi: 10.1152/jn.90606.2008

Paille, V., Fino, E., Du, K., Morera-Herreras, T., Perez, S., Kotaleski, J. H., et al. (2013). Gabaergic circuits control spike-timing-dependent plasticity. J. Neurosci. 33, 9353-9363. doi: 10.1523/JNEUROSCI.579612.2013

Pawlak, V., and Kerr, J. (2008). Dopamine receptor activation is required for corticostriatal spike-timing-dependent plasticity. J. Neurosci. 28, 2435-2446. doi: 10.1523/JNEUROSCI.4402-07.2008

Ponzi, A., and Wickens, J. (2010). Sequentially switching cell assemblies in random inhibitory networks of spiking neurons in the striatum. J. Neurosci. 30, 5894-5911. doi: 10.1523/jneurosci.5540-09.2010

Ponzi, A., and Wickens, J. (2013). Optimal balance of the striatal medium spiny neuron network. PLoS Comput. Biol. 9:e1002954. doi: 10.1371/journal.pcbi.1002954
Rabinovich, M., Volkovskii, A., Lecanda, P., Huerta, R., Abarbanel, H. D. and Laurent, G. (2001). Dynamical encoding by networks of competing neuron groups: winnerless competition. Phys. Rev. Lett. 87:068102. doi: 10.1103/PhysRevLett.87.068102

Rempel-Clower, N., and Barbas, H. (2000). The laminar pattern of connections between prefrontal and anterior temporal cortices in the rhesus monkey is related to cortical structure and function. Cereb. Cortex 10, 851-865. doi: $10.1093 /$ cercor/10.9.851

Shepherd, G. (2013). Corticostriatal connectivity and its role in disease. Nat. Rev. Neurosci. 14, 278-291. doi: 10.1038/nrn3469

Strong, S., Korberle, R., de Ruyter van Steveninck, R., and Bialek, W. (1998). Entropy and information in neural spike trains. Phys. Rev. Lett. 80, 197. doi: 10.1103/PhysRevLett.80.197

Vincent, J. L., Patel, G. H., Fox, M. D., Snyder, A. Z., Baker, J. T., Van Essen, D. C., et al. (2007). Intrinsic functional architecture in the anaesthetized monkey brain. Nature 447, 83-86. doi: 10.1038/nature05758

von Economo, C. (1929). Die Encephalitis Lethargica, ihre Nachkrankheiten und Ihre behandlung. Berlin: Urban und Schwarzenberg.

Watabe-Uchida, M., Zhu, L., Ogawa, S., Vamanrao, A., and Uchida, N. (2012). Whole-brain mapping of direct inputs to midbrain dopamine neurons. Neuron 74, 858-873. doi: 10.1016/j.neuron.2012.03.017

Zheng, P., and Triesch, J. (2014). Robust development of synfire chains from multiple plasticity mechanisms. Front. Comput. Neurosci. 8:66. doi: $10.3389 /$ fncom. 2014.00066

Conflict of Interest Statement: The author declares that the research was conducted in the absence of any commercial or financial relationships that could be construed as a potential conflict of interest.

Copyright (c) 2016 Kozloski. This is an open-access article distributed under the terms of the Creative Commons Attribution License (CC BY). The use, distribution or reproduction in other forums is permitted, provided the original author(s) or licensor are credited and that the original publication in this journal is cited, in accordance with accepted academic practice. No use, distribution or reproduction is permitted which does not comply with these terms. 